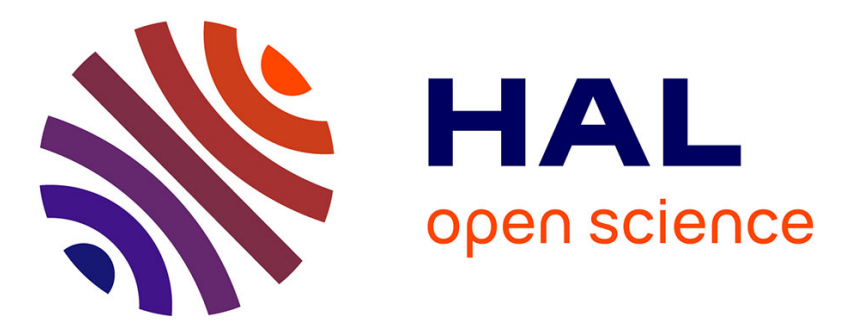

\title{
Multi-frequency Electromagnetic Sounding of the Galilean Moons
}

\author{
Mario Seufert, Joachim Saur, Fritz M. Neubauer
}

\section{To cite this version:}

Mario Seufert, Joachim Saur, Fritz M. Neubauer. Multi-frequency Electromagnetic Sounding of the Galilean Moons. Icarus, 2011, 214 (2), pp.477. 10.1016/j.icarus.2011.03.017 . hal-00786869

\section{HAL Id: hal-00786869 \\ https://hal.science/hal-00786869}

Submitted on 11 Feb 2013

HAL is a multi-disciplinary open access archive for the deposit and dissemination of scientific research documents, whether they are published or not. The documents may come from teaching and research institutions in France or abroad, or from public or private research centers.
L'archive ouverte pluridisciplinaire $\mathbf{H A L}$, est destinée au dépôt et à la diffusion de documents scientifiques de niveau recherche, publiés ou non, émanant des établissements d'enseignement et de recherche français ou étrangers, des laboratoires publics ou privés. 


\section{Accepted Manuscript}

Multi-frequency Electromagnetic Sounding of the Galilean Moons

Mario Seufert, Joachim Saur, Fritz M. Neubauer

PII:

S0019-1035(11)00108-4

DOI:

10.1016/j.icarus.2011.03.017

Reference:

YICAR 9760

To appear in: $\quad$ Icarus

Received Date: $\quad 21$ September 2010

Revised Date: $\quad 18$ February 2011

Accepted Date: $\quad 16$ March 2011

Please cite this article as: Seufert, M., Saur, J., Neubauer, F.M., Multi-frequency Electromagnetic Sounding of the Galilean Moons, Icarus (2011), doi: 10.1016/j.icarus.2011.03.017

This is a PDF file of an unedited manuscript that has been accepted for publication. As a service to our customers we are providing this early version of the manuscript. The manuscript will undergo copyediting, typesetting, and review of the resulting proof before it is published in its final form. Please note that during the production process errors may be discovered which could affect the content, and all legal disclaimers that apply to the journal pertain. 


\title{
Multi-frequency Electromagnetic Sounding of the Galilean Moons
}

\author{
Mario Seufert*,a, Joachim Saur ${ }^{\mathrm{a}}$, Fritz M. Neubauer ${ }^{\mathrm{a}}$ \\ ${ }^{a}$ University of Cologne, Institute of Geophysics and Meteorology, Zuelpicher Str. $49 a$ \\ D-50923 Cologne, Germany
}

\section{Abstract}

Induced magnetic fields provide the unique possibility to sound the conductive interior of planetary bodies. Such fields are caused by external time-variable magnetic fields. We investigate temporal variations of the Jovian magnetospheric field at multiple frequencies at the positions of the Galilean moons and analyze possible responses due to electromagnetic induction within multi-layered interior models of all four satellites. At the Jovian satellites the magnetic field varies with the synodic rotation period of Jupiter's internal field (about 10 hours), fractions of this period (e.g., 1/2 and $1 / 3$ ) due to higher order harmonics of the internal field, the orbital periods of the satellites ( $\sim 40$ hours at Io to $\sim 400$ hours at Callisto) and the solar rotation period (about 640 hours) and its harmonics due to variabilities of the magnetopause field. To analyze these field variations, we use a magnetospheric model that includes the Jovian internal field, the current sheet field and fields due to the magnetopause boundary currents. With this model we

\footnotetext{
*Corresponding Author

Email addresses: seufert@geo.uni-koeln.de (Mario Seufert), saur@geo . uni-koeln. de (Joachim Saur), neubauer@geo.uni-koeln. de (Fritz M. Neubauer)
} 
calculate magnetic amplitude spectra for each satellite orbit. These spectra provide the strengths of the inducing signals at the different frequencies for all magnetic components. The magnetic fields induced in the interiors of the satellites are then determined from response functions computed for different multi-layer interior models including conductive cores and ocean layers of various conductivities and thicknesses. Based on these results we discuss what information about the ocean and core layers can be deduced from the analysis of induction signals at multiple frequencies. Even moderately thick and conductive oceans produce measurable signal strengths at several frequencies for all satellites. The conductive cores cause signals which will be hardly detectable. Our results show that mutual induction occurs between the core and the ocean. We briefly address this effect and its implications for the analysis of induced field data. We further note that close polar orbits are preferable for future Jupiter system missions to investigate the satellites interiors.

Key words: Jupiter, satellites, Magnetic fields, Interiors, Magnetosphere

\section{Introduction}

$2 \quad$ Jupiter's major satellites harbor a unique variety of scientific treasures 3 which can be investigated through electromagnetic induction signals. There is, for example, substantial evidence for the existence of a liquid water ocean beneath the surface of Europa (e.g., Khurana et al., 1998, Neubauer, 1998b,

6 Kivelson et al., 2000, Zimmer et al., 2000, Schilling et al., 2007, Anderson 7 et al., 1998, Spohn and Schubert, 2003). Several separate lines of scientific 8 evidence led to this conclusion. One indicator were surface features inter- 
preted as cracks of a layer of ice above a layer of liquid water (Carr et al., 1998, Pappalardo et al., 1999). Radio Doppler measurements suggest a differentiated interior of Europa with a top icy layer of about 80 to 170 kilometers thickness (Anderson et al., 1998). According to several thermal models this layer might be at least partially molten (e.g., Schubert et al., 1986, Spohn and Schubert, 2003). These facts are, however, only indirect evidence for the existence of an ocean. The strongest indicator for the present-day existence of subsurface oceans was provided by magnetometer measurements of the Galileo spacecraft. These measurements showed signatures of a magnetic field induced inside the satellite which could only be explained by the existence of a conductive subsurface ocean but not by less conductive ice (Khurana et al., 1998, Neubauer, 1998a, Kivelson et al., 2000, Zimmer et al., 2000, Volwerk et al., 2007). Similar observations have been made at Callisto (Khurana et al., 1998, Neubauer, 1998a, Kivelson et al., 1999, Zimmer et al., 2000) making it another candidate for harboring liquid water. Yet another subsurface water ocean might be located inside Ganymede, the largest of the Galilean moons (Kivelson et al., 2002). Additionally, Ganymede is the only known satellite in our solar system which possesses an intrinsic magnetic field (Kivelson et al., 1996, Jia et al., 2009). Therefore, information about its highly likely conductive core would be of great value. For Io some authors proposed the existence of an at least partially molten magma ocean (Keszthelyi et al., 1999). Khurana et al. (2009) recently suggested that magnetic measurements at Io may also include signatures of fields induced in this ocean. All of the features mentioned above present subsurface layers of enhanced conductivity. Information about these layers can therefore be gained 
through studies of the electromagnetic induction at the Galilean moons.

The theoretical base for the analysis of induced fields and the determination of the interior electrical conductivity of a planetary body was developed for scientific problems at the earth (e.g., Parkinson, 1983, Schmucker, 1985, Olsen, 1999). In principle any conductive body exposed to a time-variable external magnetic field gives rise to an induced field. For a stratified body the amplitude and phase of the induced field (secondary field) relative to the inducing field (primary field) depend on the conductivity and thicknesses of the interior layers (Parkinson, 1983). Generally an infinite set of internal structures may explain the same secondary field signal at one particular frequency. However, by a combined analysis of signals caused by a primary field varying at multiple frequencies it is possible to reduce the set of valid conductivities and shell thicknesses (Khurana et al., 2002, Saur et al., 2010). Low frequencies are of special interest here as they penetrate deeper into conductive layers (Parkinson, 1983, Saur et al., 2010).

At the Galilean moons the time-variable primary field is provided by the Jovian magnetosphere (e.g., Neubauer, 1999, Saur et al., 2010). In the satellites' vicinity magnetic field measurements additionally include contributions from the induced fields and fields caused by the interaction of the satellite with the surrounding magnetospheric plasma (Schilling et al., 2008, Saur et al., 2010) . The main uncertainty for the interpretation of the total field comes from the plasma interaction. Extensive numerical modeling is necessary to determine the contribution from this field (Schilling et al., 2007). A detailed description of the plasma interaction is beyond the scope of this paper. However, we briefly address the role of the plasma interaction fields in 
section 4.3. In the following we focus on a prediction of the magnetospheric background field and the secondary fields caused by its temporal variations.

Previous studies of the inductive response of the Galilean moons (e.g., Zimmer et al., 2000, Khurana et al., 2002, Schilling et al., 2007) predominantly consider the rotation period of Jupiter with some smaller discussion of the orbital periods. We present the first systematic search for available frequencies and the corresponding amplitudes of the Jovian magnetospheric field at the position of all Galilean moons (sections 2 to 4 ). We determine low frequency fluctuations suitable for deep sounding of the satellites' interiors. In the second part of our analysis (sections 5 to 6 ) we examine the inductive response of the satellites on the basis of several interior models. Instead of the simple one or two layer models used by other authors (e.g., Zimmer et al., 2000, Schilling et al., 2007) we apply realistic multi-layer models to determine the response functions. We investigate whether secondary fields of the satellites' oceans or conductive cores will be measurable for some of the available frequencies. In the presence of multiple conductive layers the secondary field of one of these layers may in turn induce another magnetic field. We discuss this mutual induction effect and its implication for the analysis of magnetic field measurements in section 6.5. In section 7 we determine preferable flyby conditions for the measurement of induced fields at future Jovian system missions. We start with a short description of the sources of frequencies of the magnetospheric field available for induction studies. 


\section{Available frequencies}

There are several physical processes which lead to different periodicities of the magnetospheric field at the Galilean moons. Most of the magnetic fluctuations are not caused by changes of the field itself, but by underlying movements of the field structure relative to the satellites due to celestial mechanics. In this section we discuss their origin and separate them regarding the corresponding celestial period.

\subsection{Jupiter's rotation period}

The major magnetic field variability at the Galilean moons originates from the $9.6^{\circ}$ tilt of the internal field of Jupiter with respect to the Jovian spin axis. As Jupiter and its field rotate faster (every 10 hours) than the satellites orbital period (about 40 to $400 \mathrm{~h}$ ), the satellites are alternately positioned above and below the Jovian magnetic equator. This change of position in the field causes magnetic variations at the satellites' positions. The period of these fluctuations is the synodic rotation period of Jupiter in the rest frame of the satellite. Additionally, temporal variations with the same period are caused by the rotation of the current sheet magnetic field. The current sheet in the inner magnetosphere is roughly aligned with the Jovian magnetic equator and mainly corotates with the internal field. The distance of the satellites to the center of the sheet changes periodically as well. This results in magnetic fluctuations with the synodic rotation period of Jupiter which add to the internal field fluctuations. Additionally, higher order harmonics of the internal field and the current sheet field lead to fluctuations at whole-number fractions of the synodic rotation period. The Jovian rotation therefore causes 
a variety of inducing magnetic variations with periods of slightly above $10 \mathrm{~h}$, 5 h, 3.3 h etc.

\subsection{The orbital periods of the satellites}

Another set of periodicities has its origin in the movement of the satellites themselves. The inclination and eccentricity of the satellites' orbits lead to a periodic change of latitude and distance with respect to the internal field. The corresponding magnetic fluctuations encountered by the satellite have the period of its rotation (from about $40 \mathrm{~h}$ at Io to $400 \mathrm{~h}$ at Callisto). The satellites' motions relative to the magnetopause structure also cause temporal variations with the rotational periods of the satellites. At the day side of the magnetosphere the satellites are much closer to the source region of the magnetopause field than on the night side. The difference between the closest approach and the maximum distance to the magnetopause determines the amplitude of the resulting magnetic fluctuation.

\subsection{The solar rotation period}

The magnetospheric field and the magnetopause structure constantly change due to the highly variable solar wind pressure. Periodic variations of the solar wind ram pressure, which are sufficiently slow to allow a contraction or expansion of the magnetosphere, lead to reactions which resemble a "breathing" of the whole magnetospheric structure.

This movement of the magnetopause field's source region leads to magnetic fluctuations at the satellites. One such periodicity of the solar wind strength is the solar rotation period (about $640 \mathrm{~h}$ ) and harmonics of this period. 


\subsection{Other periodicities}

There are possibly several additional fluctuations to the ones described above. A temporal variability of the volcanism of Io, for example, would influence the strength of the current sheet field. Such changes in the activity of Io's volcanism have been reported recently by Rathbun and Spencer (2010). It is, however, not clear to what extend these fluctuations influence the magnetospheric field of the Jovian system. It may also be possible to use aperiodic variations of the magnetospheric field for induction studies. For example, the variation of the magnetopause field during a solar storm could provide a suitable excitation, especially at Callisto. Long term variabilities of the solar wind, for example due to the 11-year solar cycle, may also lead to inducing fields arising from the magnetopause. Finally, slow changes of the orbital elements like the drift of the argument of the perihelion or changes of the eccentricity or inclination can lead to minor magnetic field fluctuations over very long periods. To detect such weak, very low frequency signals is, however, rather unrealistic, as it would be necessary to perform measurements over a period of several decades. All periods mentioned above are long enough to sound deep into the satellites' interiors. Periodicities with much shorter periods could, however, still be used to examine the regional or even local crustal structures within a few kilometers below the surface if the chosen conductivities are realistic. For unexpectedly low electric conductivities such short periods may even be necessary to probe the deep interiors. This may be the case at Ganymede, where the magnitude of the induction signature is not quite clear. The most important source for such signals could be magnetospheric, i.e. the Jovian counterpart of ULF waves or pulsations in 


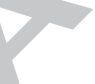

the nomenclature of geomagnetism (e.g. Krupp et al., 2004, Glassmeier et al., 2004). These waves are triggered for example by the Kelvin-Helmholtz instability, the drift-mirror instability or ion cyclotron instabilities towards shorter periods. For example, long periods of 40 minutes (Wilson and Dougherty, 2000, e.g.) have been observed in large regions of the Jovian magnetosphere as well as periods of $800 \mathrm{~s}$ and $1200 \mathrm{~s}$ associated with the Io torus (Glassmeier et al., 1989).

\section{Magnetosphere Model}

The Jovian magnetosphere is a complex system controlled by many physical effects which are addressed by different models. In our simulations we include models for the Jovian internal field, the magnetospheric current sheet and the magnetopause field. By analyzing the resulting field of each model separately we can determine the inducing fields of each part of the magnetosphere. The total magnetospheric field can be gained from a superposition of the separate model results.

\subsection{Jupiter's internal field}

The field generated by the internal dynamo of Jupiter $\mathbf{B}_{\text {INT }}$ can be described by a scalar potential $\mathrm{U}_{\text {INT }}$ of the form (e.g., Chapman and Bartels, 1940):

$$
\mathrm{U}_{\mathrm{INT}}=R_{J} \sum_{l=1}^{\infty}\left(\frac{R_{J}}{r}\right)^{l+1} \sum_{m=0}^{l} P_{l}^{m}(\cos \theta)\left[g_{l}^{m} \cos (m \Phi)+h_{l}^{m} \sin (m \Phi)\right] .
$$

In this expression $R_{J}$ is the radius of Jupiter $(71,398 \mathrm{~km}), P_{l}^{m}$ are the Schmidt-normalized Legendre functions of degree $l$ and order $m$ and $g_{l}^{m}$ and 
$h_{l}^{m}$ are the Schmidt coefficients of the field. The coordinate system is defined by the radial distance to Jupiter $r$, the colatitude $\theta$ and the longitude $\Phi$. The internal magnetic field follows from:

$$
\mathbf{B}_{\mathrm{INT}}=-\nabla \mathrm{U}_{\mathrm{INT}}
$$

Several authors have analyzed data from various spacecraft to determine the magnetic field coefficients $g_{l}^{m}$ and $h_{l}^{m}$ of Jupiter. In this study we use the VIP4 model by Connerney et al. (1998). The coefficients of this model were fit to Pioneer and Voyager data and observations for the location of Io's footprint in the Jovian auroral oval. We include coefficients up to $m, l=3$ in our analysis. Higher order coefficients are neglected as they are too uncertain and too weak at the satellites positions. Therefore, this study only considers the dipole, quadrupole and octopole parts of the field.

\subsection{The current sheet field}

The second contribution to the magnetospheric field, which we take into account is the field arising from the magnetospheric current sheet. This sheet of plasma is located near the magnetic equator and bends toward the rotational equator with increasing distance to Jupiter. The plasma particles drift due to the internal field of Jupiter and the centrifugal force and generate an additional magnetic field. This current sheet field $\mathbf{B}_{\mathrm{CS}}$ has been modeled by Khurana (1997). They used an Euler potential description in cylindrical coordinates $(\rho, \phi$ and $z$ ) relative to the dipole equator of the following form:

$$
\mathbf{B}_{\mathrm{CS}}=\nabla f(\rho, \phi, z) \times \nabla g(\rho, \phi, z),
$$


with

$$
\begin{aligned}
& f=-C_{1} \rho\left[\tanh \left(\frac{r_{01}}{r}\right)\right]^{a_{1}} \ln \cosh \left(\frac{z-z_{c s}}{D_{1}}\right) \\
& +\int \rho\left\{C_{2}\left[\tanh \left(\frac{\rho_{02}}{\rho}\right)\right]^{a_{2}}+C_{3}\left[\tanh \left(\frac{\rho_{03}}{\rho}\right)\right]^{a_{3}}+C_{4}\right\} \mathrm{d} \rho
\end{aligned}
$$

and

$$
g=\phi+p\left[1+q \tanh ^{2}\left(\frac{z-z_{c s}}{D_{2}}\right)\right] \rho .
$$

Here $z_{c s}$ represents the current sheet's distance from the magnetic equator defined as:

$$
z_{c s}=\rho \tan \left(9.6^{\circ}\right)\left[\frac{x_{0}}{x} \tanh \left(\frac{x}{x_{0}}\right) \cos (\phi-\delta)-\cos (\phi-\pi)\right],
$$

with

$$
\delta=\pi-\frac{\Omega_{J} \rho_{0}}{v_{0}} \ln \cosh \left(\frac{\rho}{\rho_{0}}\right) .
$$

The parameters $C_{1}$ to $C_{4}, a_{1}$ to $a_{3}, D_{1}, D_{2}, r_{01}, \rho_{0}, \rho_{02}, \rho_{03}, x_{0}, q$ and $p$ of these expressions were fit to various spacecraft data to reproduce the structure of the sheet. We used the so called common model given by Khurana (1997) which presents the best fit to multiple data sets. The calculated current sheet field needs to be transformed from the cylindrical coordinate system to the same coordinates used for the other models.

\subsection{The magnetopause field}

The third part of the magnetospheric field which we consider is the magnetic source region of the magnetopause. The magnetopause field is generated by Chapman-Ferraro currents (see Chapman and Ferraro, 1930) flowing in the boundary layer given by the pressure equilibrium between the solar wind 
pressure and the sum of the magnetic pressure and plasma pressure of the magnetosphere. A global model for this field was derived by Engle (1992). We chose this model over others (e.g. by Alexeev and Belenkaya, 2005) as it is easy to implement and can be used to describe a time-variable magnetopause structure. Following the approach of Engle and Beard (1980), Engle (1992) calculated the surface of the boundary layer from the pressure balance of a magnetosphere model with the solar wind. Their magnetospheric field included the current sheet model of Connerney et al. (1981) and a simple dipole field. The surface calculations were used to fit coefficients of a scalar potential $\mathrm{U}_{\mathrm{MP}}$ which we use to describe the magnetopause field $\mathbf{B}_{\mathrm{MP}}$ :

$$
\begin{gathered}
\mathbf{B}_{\mathrm{MP}}(r, \theta, \Phi)=-\mathrm{C}_{n} \nabla \mathrm{U}_{\mathrm{MP}}(r, \theta, \Phi), \\
\mathrm{U}_{\mathrm{MP}}=\sum_{l=1}^{l_{\max }}\left(\frac{r}{R_{s s}}\right)^{l} \sum_{m=0}^{l} G_{l}^{m} P_{l}^{m}(\cos \theta) \cos (m \Phi) .
\end{gathered}
$$

Here $R_{s s}$ is the magnetopause subsolar point distance and $\mathrm{C}_{n}$ a normalization constant which is defined by the magnetospheric field strength (internal field and current sheet field) at this distance. By adjusting $\mathrm{C}_{n}$ it is possible to scale the model to different locations for $R_{s s}$ representing changing solar wind conditions. As Engle (1992) explicitly used the Connerney et al. (1981) model to describe the current sheet, we also applied this model instead of the Khurana (1997) model to determine $\mathrm{C}_{n}$ in order to follow their approach as closely as possible. Please note, that the intention of this paper is to give a realistic estimate for the strength of the primary field at each frequency. Therefore, we use the more realistic description of Khurana (1997) to determine the contributions by the current sheet, while the model by Connerney 
et al. (1981) is used to determine the field at $R_{s s}$ in the context of the magnetopause model. Because each of the model results are analyzed separately there is no inconsistency in using different models here. We chose a default subsolar point distance of $60 R_{J}$.

Engle (1992) gave coefficients $G_{l}^{m}$ for three different orientations of the magnetic axis of Jupiter, $\alpha=0^{\circ}$ (tilted toward the Sun), $\alpha=180^{\circ}$ (tilted away from the Sun) and $\alpha=90^{\circ}$ (no tilt). Bode (1994) additionally derived a functional expression of these coefficients for arbitrary tilt configurations. Our analysis was carried out for each of these models. There were, however, only negligible differences in the calculated inducing fields. We therefore only present results for the $\alpha=90^{\circ}$ model in this paper.

Figure 1 illustrates the field lines of the models used in this paper. Figure 1A shows the confined structure of the current sheet of the Khurana (1997) model combined with the VIP4 model. The vertical sweep back of the field lines can easily be noticed. As the magnetopause model is not consistent with the Khurana (1997) model, it is displayed separately in combination with the Connerney et al. (1981) current sheet field (figure 1B) and the VIP4 model. The magnetopause boundary is clearly visible around $60 \mathrm{R}_{J}$.

\subsection{Variability of the magnetopause}

The position of the magnetopause is controlled by the highly variable solar wind. As mentioned in section 2, periodicities in the solar wind can lead to fluctuations of the magnetopause which in turn give rise to temporally varying inducing fields in the Jovian system.

To simulate this effect we make use of the possibility to scale the Engle (1992) model to various magnetopause distances. Based on a study of Voy- 
ager data by Huddleston et al. (1998), Alexeev and Belenkaya (2005) gave an empirical fit of the magnetopause position $R_{s s}$ with respect to the pressure of the solar wind $p_{s w}$ :

$$
R_{s s}=\frac{35.5 R_{J}}{p_{s w}^{0.22}[n P a]} .
$$

This relationship can be used to compute a time series of subsolar point distances from solar wind data. The Engle (1992) model can then be scaled using this time series to simulate the variability due to the solar wind.

For our simulation, we used solar wind data collected by the Ulysses spacecraft which were provided by the Planetary Data System. The solar wind ram pressure can be calculated from the velocity and density of the plasma. Ulysses did not monitor the actual plasma conditions right outside the Jovian magnetosphere. However, these data sets which were recorded near Jupiter's orbit at 5.0 AU to 5.5 AU over a longer period of time. Since the absolute timing of the solar wind fluctuations is irrelevant for our analysis, we did not extrapolate the data spatially to the exact position of Jupiter. Our data processing included an averaging of the data over $2 \mathrm{~h}$. We analyzed Ulysses data sets of the years 1992, 1997 to 1999 and 2003 to 2005. The strongest variations were encountered during the second half of 1992. In this paper we therefore present results of magnetic fluctuations predicted for this time series.

The velocity time series (Figure 2A) clearly show variations with the solar rotation period ( $\approx 27$ days). It is harder to see this periodicity in the resulting values for $R_{s s}$ calculated from equation 10 . The reason for this is the density which does not show such a clear periodicity but varies 
over a much greater range. Figure 2B shows the computed magnetopause subsolar point distances which span between 40 and 100 Jovian radii with an average of about $70 R_{J}$. These values lie in the typical range for $R_{s s}$ at Jupiter (Huddleston et al., 1998). The short-lived peaks exceeding distances of $100 R_{J}$ might be unrealistic but have no significance for our analysis. They only cause a slight decrease of the already rather weak magnetopause field at the satellites for these $R_{s s}$ distances.

\subsection{Analysis procedure}

To distinguish the influence of different magnetospheric and orbital aspects at the Galilean moons we performed a series of simulations with different setups. Our model results were computed using time series for the satellites' positions in System III coordinates for simple circular orbits at the satellites' mean distance to Jupiter and orbits which include the satellites' inclination and eccentricity, respectively (values given in table 1). Additionally, different internal field and magnetopause configurations were considered. The internal field was determined for coefficients of degree and order 1, 2 or 3 , respectively, to distinguish the contributions of the different magnetic moments. The magnetopause field was simulated for a fixed value of $R_{s s}=$ $60 R_{J}$. For all of these simulations we used time series with a length of 1000 Jovian rotations, sampled with 50,000 data points. Additionally, to simulate the magnetopause variability the Engle (1992) model was dynamically scaled to the varying subsolar point distances. For this part of the analysis we had to use a limited length of our time series which follows from the suitable Ulysses data. It covers about 517 rotations with 41,000 data points. The results of these simulations are time series for all magnetic field components. A Fast 
Fourier Transform (FFT) analysis of the results provides us with amplitude spectra for the inducing fields at all the satellites. As these spectra mainly consist of delta peaks it was necessary to suppress spectral leakage in order to obtain sufficiently precise amplitude values. We did this for each resulting delta peak separately, by repeating the analysis with a slightly different length of our generic time series until we obtained a maximum amplitude value. The resulting amplitudes and underlying frequencies for the models presented in this section and for a time-variable magnetopause are discussed in section 4.

\section{Results for the Primary Field}

\subsection{The magnetospheric field at the Galilean moons}

The models presented in section 3 can be used to compute the magnetic fields along the orbits of Io, Europa, Ganymede and Callisto. Figure 3 shows the variations of $\mathrm{B}_{r}$ and $\mathrm{B}_{\Phi}$ (in System III coordinates) at each satellite during a simulation time of 1000 Jovian rotations. The field is elliptically polarized with one cycle representing one synodical rotation of Jupiter with respect to the satellite. At Callisto this polarization becomes nearly linear. The strength of the temporal field variations decreases from $\left(\mathrm{B}_{r}, \mathrm{~B}_{\Phi}\right)$ $=(650 \mathrm{nT}, 370 \mathrm{nT})$ at Io to about $\left(\mathrm{B}_{r}, \mathrm{~B}_{\Phi}\right)=(40 \mathrm{nT}, 10 \mathrm{nT})$ at Callisto. These are the amplitudes of the major inducing signals at the synodic Jovian rotation period. The orbital eccentricities and inclinations lead to some broadening of the lines in figure 3, especially for Europa where these values are highest (see table 1). Compared to the strong variations of the other field components $\mathrm{B}_{\theta}$ is almost constant. There are, however, several minor 
temporal variations which affect the $\mathrm{B}_{\theta}$ component. The spectral analysis presented in the section 4.2 discusses the temporal variations of all components in greater detail.

\subsection{Periods and amplitudes of the inducing field}

We analyzed amplitude spectra of the magnetic field time series for the periods discussed in section 2. The spectra of a superposition of the magnetic fields of all our models, for inclined and eccentric orbits and for a time-variable magnetopause are shown in figure 4. Table 2 lists all significant (arbitrarily defined as $>0.2 \mathrm{nT}$ ) peak amplitudes and periods. These values have been derived from separate spectra for each magnetospheric contribution and magnetic component. The associated sources are indicated in the last column of the table. Small discrepancies between the amplitudes shown in figure 4 and the more accurate values in table 2 can be caused by spectral leakage at some peaks in the total spectrum.

\subsubsection{Inducing fields with Jupiter's rotational period}

The major spectral peaks in figure 4 can be found in the $\mathrm{B}_{r}$ and $\mathrm{B}_{\Phi}$ components at the synodical rotation period of Jupiter with respect to the satellites. The exact periods are $12.95 \mathrm{~h}$ for Io, $11.23 \mathrm{~h}$ for Europa, $10.53 \mathrm{~h}$ for Ganymede and $10.18 \mathrm{~h}$ for Callisto. The $\mathrm{B}_{r}$ amplitudes are quite large for all of the satellites. They reach up to $750 \mathrm{nT}$ at Io, $215 \mathrm{nT}$ at Europa and about $85 \mathrm{nT}$ at Ganymede. Even at Callisto, were the dipole field is already quite weak but the current sheet variability becomes more important, we predict amplitudes of about $40 \mathrm{nT}$. The $\mathrm{B}_{\Phi}$ component provides additional inducing signals of $370 \mathrm{nT}$ at Io, $105 \mathrm{nT}$ at Europa, $30 \mathrm{nT}$ at Ganymede and 
$10 \mathrm{nT}$ at Callisto. The inducing fields in $\mathrm{B}_{\theta}$ at this period are caused by the quadrupole moments. With values of $100 \mathrm{nT}$ at Io, $16 \mathrm{nT}$ at Europa, $2.5 \mathrm{nT}$ at Ganymede and only $0.3 \mathrm{nT}$ at Callisto they are much weaker.

The spectra in figure 4 further show inducing fields with short periods (1/2 and $1 / 3$ of the synodic Jovian rotation) evident both in $\mathrm{B}_{r}$ and $\mathrm{B}_{\Phi}$. The corresponding amplitudes of $\mathrm{B}_{r}$ drop rapidly from $108 \mathrm{nT}$ and $16 \mathrm{nT}$ at Io to about $17 \mathrm{nT}$ and $2.8 \mathrm{nT}$ at Europa and $2.6 \mathrm{nT}$ and $3.3 \mathrm{nT}$ at Ganymede. At Callisto the only significant short period contribution of $6 \mathrm{nT}$ is caused by the current sheet structure. Some rather weak inducing fields of $1 / 2$ of the synodic Jovian rotation period can also be found in $\mathrm{B}_{\theta}$.

According to our simulation results, every magnetic component provides at least two short period $(<13 \mathrm{~h})$ inducing signals for each satellite. If the interior of the satellites is sufficiently conductive the amplitude of most of these signals should be large enough to provide measurable induced signals at multiple frequencies.

\subsubsection{Inducing fields with the satellites' orbital periods}

The inclinations and eccentricities of the satellites' orbits and their changes in distance with respect to the magnetopause field cause inducing signals at the satellites' orbital period. The corresponding periods are: $42.45 \mathrm{~h}$ for Io, 85.22 h for Europa, $171.70 \mathrm{~h}$ for Ganymede and $400.55 \mathrm{~h}$ for Callisto. The orbital inclination causes inducing signals predominantly in the $\mathrm{B}_{r}$ component. Except for $10 \mathrm{nT}$ at Europa these signals are rather weak (2.9 nT at Io, $1.7 \mathrm{nT}$ at Ganymede and only $0.7 \mathrm{nT}$ at Callisto). However, the eccentricity of the orbits and the magnetopause field cause additional periodic signals in the $\mathrm{B}_{\theta}$ component. The amplitudes of these contributions add up to a 
maximum of $27 \mathrm{nT}$ at Io $17 \mathrm{nT}$ at Europa and about $2.5 \mathrm{nT}$ at Ganymede. At Callisto the amplitude of this signal is almost entirely determined by the position of the magnetopause. For a magnetopause standoff distance of $\mathrm{R}_{s s}=$ $60 \mathrm{R}_{J}$ we predict a signal of about $3 \mathrm{nT}$. The strength of this magnetopause field $\mathrm{B}_{\theta}$ peak depends on the strength of the solar wind. Figure 5 shows the dependency of the peak value on the subsolar point standoff distance in the magnetopause model. Strong solar wind conditions $\left(\mathrm{R}_{s s} \approx 40 \mathrm{R}_{J}\right)$ lead to increased amplitudes of about $6 \mathrm{nT}$ for Callisto and $4 \mathrm{nT}$ at Ganymede.

These long period inducing signals are of special interest as they penetrate deeper into the satellites' bodies. For Callisto and Ganymede we predict only weak long period signals which pose a challenge to magnetometer measurements on future Jupiter system missions.

\subsubsection{Inducing fields with the solar rotation period}

The temporal variability of the magnetopause gives rise to a third set of field periodicities. The spectra in figure 4 show peaks arising at the solar rotation period of $641.9 \mathrm{~h}$. At Callisto and Ganymede this feature is clearly visible with an amplitude of $1.2 \mathrm{nT}$. For Io and Europa the peaks are of about the same strength but small relative to other features in $\mathrm{B}_{\theta}$. The reason for the similar strength at all satellites is that the solar rotation period is longer than their orbital period. Averaged over multiple orbits all satellites can be assumed to be approximately at the location of Jupiter. They therefore experience roughly the same variations regardless of their orbital distance.

These very long period inducing signals are again quite weak and will be rather difficult to measure. However, they pose the opportunity to sound very deeply into the satellites' interiors. 


\subsection{Plasma interaction fields}

The main factors reducing the detectability of the signals presented above are the plasma interaction fields which occur in the vicinities of the satellites. In the last row of table 2 we give an estimate of the maximum interaction fields near the satellites. The maximum total current in both Alfvén wings due to the interaction is given after Neubauer (1980, 1998b) and Saur (2004) as:

$$
I=8 v_{0} B_{0} R_{s a t} \Sigma_{A} \overbrace{\frac{\Sigma_{P}}{\Sigma_{P}+2 \Sigma_{A}}}^{\alpha},
$$

where we assume the plasma velocity $\mathbf{v}_{0}$ to be perpendicular to the magnetic field. The values in table 2 are calculated for the maximum current $\mathrm{I}_{\max }$, i.e. in the limit of an infinite ionospheric Pedersen conductance $\Sigma_{P}(\alpha=1)$ and for maximum values of the velocity $\mathrm{v}_{0}$, the background magnetic field $\mathrm{B}_{0}$ and the Alfvén conductance $\Sigma_{A}$ given by Kivelson et al. (2004). The magnetic field due to the plasma interaction, $\mathrm{B}_{p}$, follows from the current flowing through the satellites' ionospheres (with an assumed ionospheric extension of $300 \mathrm{~km})$ :

$$
\oint B_{p} d x=\mu_{0} I_{\text {max }} \Rightarrow B_{p}=\mu_{0} \frac{I_{\max }}{\pi\left(R_{\text {sat }}+300 k m\right)} .
$$

The values for the interaction given in table 2 should be taken as a crude estimate of the maximum possible plasma fields occurring in addition to the induced fields examined in this paper. Examples for field perturbations of this strength (700 nT at Io and $300 \mathrm{nT}$ for Europa) are the I0 (Kivelson et al., 1996b) and the E12 (Kivelson et al., 2000) flybys of the Galileo spacecraft. 
Most of the time, however, the plasma interaction is much weaker. While the values in table 2 (as well as the I0 and E12 flybys) correspond to situations when the satellites are in the center of the current sheet, the interaction strength significantly diminishes as the satellites move away from this center. For example during the Europa E14 and Callisto C3 encounters of Galileo discussed by Zimmer et al. (2000) the field perturbations were as low as $20 \mathrm{nT}$ and $5 \mathrm{nT}$, respectively. One reason for this dependence is the Alfvén conductance which decreases by a factor of up to $1 / 2$ for Io, $1 / 5$ for Europa, 1/10 for Ganymede and 1/250 for Callisto (Kivelson et al., 2004) with distance from the sheet's center. The plasma interaction field $\mathrm{B}_{P}$ decreases accordingly. Additionally, the factor $\alpha$ in equation (11) generally decreases as the ionospheric conductance $\Sigma_{P}$ weakens with distance to the current sheet. The reason for this is that $\Sigma_{P}$ depends on the ionospheric plasma density, which is controlled by the density of the magnetospheric plasma. Lower values for $\Sigma_{P}$ due to variations of the distance from the center of the current sheet can weaken the interaction strength $\alpha$ in some cases about the same degree given above for the Alfvén conductance. Therefore, the analysis of induced fields in the satellites' vicinities is much easier in situations when the distance to the current sheet center is large. Another factor that supports the separation of interaction fields and induced fields is the different temporal dependency of induced fields and interaction fields. While the induced fields periodically change their sign, the steady flow of plasma within the magnetosphere leads to interaction fields which only change in strength but usually not in sign. In summary, it is important to consider the plasma interaction effects for induction studies at all satellites. Strategies which consider induction and plasma 


\section{simplifies to}

interaction self-consistently are already established and were successfully applied to Europa by Schilling et al. (2007, 2008). Using such strategies it is still realistic to distinguish even weak contributions of the induction within the magnetic signals, at least when the satellites are far away from the center of the current sheet.

\section{Induction Model}

In the following parts of this paper we will examine the inductive response of the interiors of the Galilean moons to some of the inducing signals presented above. In the theoretical description of this response we assume the satellites to be differentiated spheres. The conductivities of each of the shells can be different, but they are assumed to be constant within each layer. To describe the effects of electromagnetic induction in such a conductive sphere we follow the theoretical approach of Parkinson (1983). We start with the diffusion equation which can be derived from Ohm's, Faraday's and Ampère's law and $\nabla \cdot \mathbf{B}=0$

$$
\frac{\partial \mathbf{B}}{\partial t}=-\nabla \times\left(\frac{1}{\sigma \mu}(\nabla \times \mathbf{B})\right),
$$

where $\mathbf{B}$ is the magnetic field diffusing into a medium with an electrical conductivity of $\sigma$. For regions of spatially constant conductivity this equation

$2>$

$$
\frac{\partial \mathbf{B}}{\partial t}=\frac{1}{\sigma \mu} \Delta \mathbf{B} .
$$

The displacement currents in Ampère's law are neglected here as the conduction currents are substantially larger for the relevant materials and appropriate time scales for the induction at the Galilean moons (e.g., Saur et al., 
2010). We also neglect the implications of tidal motions inside the liquid or semi-liquid layers of the Galilean moons and assume that all conductive materials are at rest. In the following we take $\mu$ to equal the permeability of the vacuum $\mu_{0}$. This is justified for diamagnetic and paramagnetic materials discussed for the interiors of the Galilean moons with $\mu_{r} \approx 1$ (Saur et al., 2010). Let us assume the presence of a time-variable primary field $\mathbf{B}_{\text {pri }}$ which includes contributions from $\mathrm{N}$ different frequencies $\omega_{n}$ with amplitudes $\mathbf{B}_{n}$. Since equation (14) is linear in $\mathbf{B}$, the time-dependent component of its solution can be expressed as a Fourier decomposition of the form:

$$
\mathbf{B}_{p r i}=\sum_{n=1}^{N} \mathbf{B}_{n} e^{-i \omega_{n} t}
$$

We can now search for solutions of (14) for each periodicity of the field (for each $n$ ) separately.

The fields inside and outside the satellite need to be treated using different approaches. If we neglect the plasma currents outside the satellite, we can describe the magnetic field in this region by using a scalar potential of the form $\mathbf{B}_{\text {ext }}=-\nabla \mathrm{U}_{\text {ext }}$. This potential includes complex coefficients of both the primary field $B_{p r i}$ and the induced secondary field $B_{s e c}$ :

$$
\mathrm{U}_{\text {ext }}(r, \theta, \Phi)=R\left[B_{\text {pri }}\left(\frac{r}{R}\right)^{l}+B_{\text {sec }}\left(\frac{R}{r}\right)^{l+1}\right] S_{l}^{m}(\theta, \Phi) e^{-i \omega t}
$$

Here $R$ is the satellite's radius and $l$ and $m$ the degree and order of the spherical harmonics $S_{l}^{m}$ characterizing the potential. To describe the field inside the conducting sphere (where $\mathbf{j} \neq 0$ ) it is useful to use the vector potential $\mathbf{A}_{\text {int }}$ with $\nabla \times \mathbf{A}_{\text {int }}=\mathbf{B}_{\text {int }}$. This potential can be separated in a 
toroidal part $\mathrm{T}$ and a poloidal part $\mathrm{P}$ :

$$
\mathbf{A}_{i n t}=\mathrm{Tr}+\nabla \mathrm{P} \times \mathbf{r}
$$

We can neglect $\mathrm{T}$ as it has no radial component and therefore can neither be induced by external fields nor be detected outside the sphere (see Parkinson, 1983). When we assume a spherically symmetric conductivity distribution $(\sigma=\sigma(r))$ we can write $\mathrm{P}$ as:

$$
\mathrm{P}=\gamma F(r) S_{l}^{m}(\theta, \Phi) e^{-i \omega t}
$$

Here $F$ is a function of $r$, and $\gamma$ an additional constant both of which need to be determined. As the magnetic field $\mathbf{B}_{i n t}$, which follows from $\mathbf{A}_{\text {int }}$, has to solve the diffusion equation (14) it can be shown that $F(r)$ obeys the modified spherical Bessel equation which has two independent solutions:

$$
\begin{aligned}
& F_{1}(r, k)=\sqrt{\frac{\pi}{2 r k}} I_{l+\frac{1}{2}}(r k), \\
& F_{2}(r, k)=\sqrt{\frac{\pi}{2 r k}} K_{l+\frac{1}{2}}(r k) .
\end{aligned}
$$

These are the spherical modified Bessel functions of the first and second kind (e.g. Parkinson, 1983, Abramowitz and Stegun, 1964, Riley et al., 2006). They depend on $r$ and on the complex wave number $k=\sqrt{-i \omega \mu_{0} \sigma}$. Let us now consider the case of a sphere consisting of $J$ different shells with conductivities and radii as shown in figure 6.

Instead of explicitly deriving a solution for $\mathbf{B}$ we can formulate a response function which gives the amplitude ratio of the primary and secondary field outside the surface. We do this by solving a system of equations that follows 
from applying continuous boundary conditions for $\mathrm{B}_{r}$ and $\mathrm{B}_{\Phi}$ at the boundary between the different shells. At the surface $\mathrm{B}_{r}$ and $\mathrm{B}_{\Phi}$ need to be equal to the corresponding values of $\mathbf{B}_{\text {ext }}$. Each of the field components of $\mathbf{B}_{\text {int }}$ and $\mathbf{B}_{\text {ext }}$ depend on $S_{l}^{m}(\theta, \Phi)$ and $e^{-i \omega t}$. Therefore, these terms cancel each other. As $F_{2}$ in (20) goes to infinity at $r=0$, it is no suitable solution for the innermost shell. For all other shells with index $j$ a linear combination $C_{j} F_{1}+D_{j} F_{2}$ is the general solution. This gives rise to $2 J-1$ constants to be determined. A solution is given by the following recursive formula:

$$
\begin{aligned}
& \frac{B_{\text {sec }}}{B_{\text {pri }}}=\frac{l}{l+1} \frac{\frac{F_{1}^{\prime}\left(R k_{J}\right)}{F_{1}\left(R k_{J}\right)}-(l+1)+\frac{D_{J-1}}{C_{J-1}} \frac{F_{2}\left(R k_{J}\right)}{F_{1}\left(R k_{J}\right)}\left[\frac{F_{2}^{\prime}\left(R k_{J}\right)}{F_{2}\left(R k_{J}\right)}-(l+1)\right]}{\frac{F_{1}^{\prime}\left(R k_{J}\right)}{F_{1}\left(R k_{J}\right)}+l+\frac{D_{J-1}}{C_{J-1}} \frac{F_{2}\left(R k_{J}\right)}{F_{1}\left(R k_{J}\right)}\left[\frac{F_{2}^{\prime}\left(R k_{J}\right)}{F_{2}\left(R k_{J}\right)}+l\right]}, \\
& \frac{D_{j}}{C_{j}}=\frac{F_{1}\left(r_{j-1} k_{j}\right)}{F_{2}\left(r_{j-1} k_{j}\right)} \frac{\left(\begin{array}{c}
\frac{F_{1}^{\prime}\left(r_{j-1} k_{j}\right)}{F_{1}\left(r_{j-1} k_{j}\right)}-\frac{F_{1}^{\prime}\left(r_{j-1} k_{j-1}\right)}{F_{1}\left(r_{j-1} k_{j-1}\right)} \\
+\frac{D_{j-1}}{C_{j-1}} \frac{F_{2}\left(r_{j-1} k_{j-1}\right)}{F_{1}\left(r_{j-1} k_{j-1}\right)}\left[\frac{F_{1}^{\prime}\left(r_{j-1} k_{j}\right)}{F_{1}\left(r_{j-1} k_{j}\right)}\right. \\
\left.-\frac{F_{2}^{\prime}\left(r_{j-1} k_{j-1}\right)}{F_{2}\left(r_{j-1} k_{j-1}\right)}\right]
\end{array}\right)}{\left(\begin{array}{c}
\frac{F_{1}^{\prime}\left(r_{j-1} k_{j-1}\right)}{F_{1}\left(r_{j-1} k_{j-1}\right)}-\frac{F_{2}^{\prime}\left(r_{j-1} k_{j}\right)}{F_{2}\left(r_{j-1} k_{j}\right)}+ \\
\frac{D_{j-1}}{C_{j-1}} \frac{F_{2}\left(r_{j-1} k_{j-1}\right)}{F_{1}\left(r_{j-1} k_{j-1}\right)}\left[\frac{F_{2}^{\prime}\left(r_{j-1} k_{j-1}\right)}{F_{2}\left(r_{j-1} k_{j-1}\right)}\right. \\
\left.-\frac{F_{2}^{\prime}\left(r_{j-1} k_{j}\right)}{F_{2}\left(r_{j-1} k_{j}\right)}\right]
\end{array}\right)} \\
& \frac{D_{2}}{C_{2}}=\frac{F_{1}\left(r_{1} k_{2}\right)}{F_{2}\left(r_{1} k_{2}\right)}\left(\frac{\frac{F_{1}^{\prime}\left(r_{1} k_{2}\right)}{F_{1}\left(r_{1} k_{2}\right)}-\frac{F_{1}^{\prime}\left(r_{1} k_{1}\right)}{F_{1}\left(r_{1} k_{1}\right)}}{\frac{F_{1}^{\prime}\left(r_{1} k_{1}\right)}{F_{1}\left(r_{1} k_{1}\right)}-\frac{F_{2}^{\prime}\left(r_{1} k_{2}\right)}{F_{2}\left(r_{1} k_{2}\right)}}\right) .
\end{aligned}
$$

Here (21) represents the boundary condition at the surface, (23) at the in509 nermost shell and (22) at each shell in between. The ratio $B_{\text {sec }} / B_{\text {pri }}$ is a complex expression which can be decomposed in:

$$
\frac{B_{s e c}}{B_{p r i}}=\frac{l}{l+1} \mathrm{~A} e^{i \phi},
$$


where $\mathrm{A}$ is the relative amplitude of the secondary field with respect to the primary field. The maximum possible amplitude is $\mathrm{A}=1$. In this case the sphere acts as a perfect conductor. $\phi$ is the phase shift of the secondary signal with respect to the primary field. $l=1$ gives the case of a dipole shape field.

The inducing signal can be seen as a diffusion-wave which is damped by the conductive media of the interior. The depth at which the amplitude of the wave has decreased by a factor of e is called skin depth $\delta$. It depends on the conductivity of each separate layer and on the frequency of the propagating wave:

$$
\delta=\sqrt{\frac{2}{\sigma \mu_{0} \omega}} .
$$

With the amplitudes and the periods of the primary field determined in section 4 we can now determine the corresponding secondary field by using suitable interior conductivity structures for each satellite.

\section{Results for the Secondary Field}

We now analyze the secondary fields generated by the primary fields derived in section 4.2. Three periods of the primary field are treated separately: the synodic rotation period of Jupiter, the satellites' orbital period and the solar rotation period. For each period we apply the induction model given by equations (21) to (24) to different interior models from the literature. Two models were selected for each satellite. First we use models which explicitly assume the existence of an ocean. For Io we chose a model involving a magma ocean by Keszthelyi et al. (1999). Models considering a subsur- 
face water ocean were given by Kuskov and Kronrod (2005) for Europa and Callisto and by Kimura et al. (2009) for Ganymede. In preparation for this paper we also analyzed results from other elaborated models such as Schubert et al. (2009) for Europa and Schubert et al. (2004) or Sohl et al. (2002) for Ganymede and Callisto. The differences in the overall model setup with respect to the models presented here, mainly variations of the thicknesses of the layers, are relatively subtle from the induction point of view. Therefore, we do not provide discussion for these models. We do, however, discuss some notable differences in sections 6.3 and 6.4. We include a second, rather simple set of three layer models (consisting of crust, mantle and core) by Zhang (2003). Even though these models are less likely, they are useful to discuss the effect different features, like a smaller ice shell thickness at Europa or a larger depth of the ocean layer at Ganymede, have on the induction signals. The Zhang (2003) models also give us the opportunity to compare our results for the different satellites. In order to test the implications of different ocean configurations, we vary its thickness and conductivity over a certain range. For Io, Callisto and the Kimura et al. (2009)-Ganymede model we keep the outer crustal layer fixed and reduce the thickness of the mantle layer below the ocean as we increase its extension to preserve the satellite's radius. At Europa and for the Zhang (2003)-Ganymede model the thickness of the crust is reduced while the mantle thickness is kept fixed. The values for all other layers including the thickness of the core are kept fixed.

We present the resulting amplitudes (in \%) in contour plots covering the plausible oceans thickness and conductivity ranges (figures 7, 9, 11 and 13). In the following discussions we denominate cases which lie near the 
lower right corner of these plots as strong ocean case and cases near the upper right corner as weak ocean case. To determine the resolving power the analysis method provides for the satellite's core, we display the amplitude difference (denoted $\Delta \mathrm{A}$ in figures 7 to 13 ) between the present model and the same model without a conductive core, i.e. with a core conductivity of $10^{-9} \mathrm{~S} / \mathrm{m}$ (not exactly zero for numerical reasons). This difference gives the percentage of the core contribution to the total signal. The core influence is represented by the color plots. Note the color scales for the core influence and the isolines in black for the total induced amplitudes are two different quantities independently plotted in figures 7 to 13 . White and yellow colors indicate negative values for the core influence or regions where the amplitude decreases due to higher core conductivities. In addition to the amplitude we analyzed the phase shift of the induced signal in a similar way (figures 8, 10, 12 and 14). The relative amplitudes of the induced fields resulting from equation (24), can be multiplied with the inducing amplitudes derived in section 4.2. This yields the predicted secondary field strength which would be measurable at the surface of the satellites. Please keep in mind that all explicit values (in nT) given in this chapter should not be taken as the field strength a spacecraft could measure at flybys or in orbit around the satellite. Instead, a spacecraft at a distance $r$ would encounter fields decreased by the factor $(r / R)^{3}$ for a dipole field. This is the case for the locally homogeneous inducing fields considered in our analysis.

One major uncertainty of our approach are the values for the conductivity of the materials involved. So far there is almost no scientific information available about the electrical conductivities of materials under the physical 
conditions present inside the Galilean moons. We tried to estimate these values based on various sources in the literature. The densities of the layers are used as a proxy for the state of the material. Other factors like the temperature were not available for all models and were not included in the estimation. We preferentially use values which have been given for the interior of the earth. Table 3 lists the assumed materials, the estimated conductivities and the references.

\subsection{Io}

The interior of Io has been modeled by Keszthelyi et al. $(1999,2004)$ and Zhang (2003). So far there is no direct evidence for a global magma ocean at Io. However, Keszthelyi et al. (1999) state that due to very high temperature silicate lavas discovered at the surface, ultramafic volcanism seems to be a common property of Io. They argue that this discovery is inconsistent with a largely solid interior of Io. Instead Keszthelyi et al. (1999) suggest that the existence of a mushy magma ocean could be an explanation for the observed surface features. We adapted the four layer model of Keszthelyi et al. (1999) which includes a FeS core of medium conductivity, a relatively conductive partially molten mantle and a up to $250 \mathrm{~km}$ thick magma ocean beneath a thin crust (see table 4). The second model is given by Zhang (2003). We extended this three layer model by substituting the upper part of the mantle with a magma ocean layer (table 5). This extension may not be consistent with the results of Zhang (2003), especially for thick magma ocean layers. Results for these cases should therefore not be taken as realistic predictions but as a crude estimate for the induction at Io.

Figure 7 shows the resulting relative amplitudes at three periods of the 
primary field for both models. To assess the significance of these figures, it is interesting to note that, for large conductivities and small thicknesses of the magma ocean, the amplitude isolines become straight and follow lines of constant magma ocean conductance $(A \propto \sigma h)$.

\subsubsection{Observability of the magma ocean}

For both models the isolines in figure 7 show that at the synodic rotation period as well as the orbital period amplitudes near $90 \%$ are possible for the strong magma ocean case $(\sigma>1 \mathrm{~S} / \mathrm{m}, h>5 \mathrm{~km})$. This is nearly the perfect conductor case. For the solar rotation period the maximum amplitude is about $60 \%$. For weaker magma oceans the conductivity of the partially molten mantle prevents the amplitude to drop to $0 \%$. To obtain the actual contribution by the magma ocean to the total signal one needs to subtract the signal remaining in the case of a weak magma ocean. This gives a contribution by the magma ocean of about 65 to $80 \%$ depending on the period. At Io the partially molten mantle contributes significally to the total signal. We determine the secondary signal for mantle conductivities of $10^{-4} \mathrm{~S} / \mathrm{m}$ and $10^{-2} \mathrm{~S} / \mathrm{m}$ and find an increase of up to $20 \%$ to $25 \%$ for a period of $12.95 \mathrm{~h}$ for both models. It should be noted that at $10^{-2} \mathrm{~S} / \mathrm{m}$ the conductivity of the magma ocean drops below that of the mantle beneath. In figure 7 this causes a convergence of the isolines at $10^{-2} \mathrm{~S} / \mathrm{m}$ and a reversal in the direction of the lines for smaller conductivities. The Zhang (2003) model gives amplitudes which are about $7 \%$ weaker than those obtained with the Keszthelyi et al. (1999) model. This is a result of the higher crustal thickness in this model. An increased distance to the conducting layer results in a significant decrease of the measurable signal. Due to the strong primary signals at Io 
even a relatively weak magma ocean configuration produces significant signals at the surface of Io in the orbital and synodical frequency range. For the strong magma ocean case $(\sigma>1 \mathrm{~S} / \mathrm{m}, h>5 \mathrm{~km})$ we predict signals of up to $210 \mathrm{nT}$ at a period of $12.95 \mathrm{~h}$ and $14 \mathrm{nT}$ at $42.95 \mathrm{~h}$ occurring at the surface of Io. The amplitude for the solar rotation period will be quite weak (up to $0.4 \mathrm{nT}$ ). The strong plasma interaction field at Io will, however, make it very difficult to extract even the strongest induction signals from single flyby measurements (e.g., Kivelson et al., 2001, Saur et al., 2002).

\subsubsection{Observability of the core}

The amplitude difference between the original model and a model with a nearly non-conductive core is indicated by the color plot in figure 7 . It is intriguing why this difference becomes negative (white and yellow areas in figure 7). This is possible due to mutual induction occurring between the core and the magma ocean. This effect will be discussed in greater detail in section 6.5. The core influence for both models of Io at all periods is about $-1.8 \%$ to $2.5 \%$. If we combine these values with the primary field for a period of $12.95 \mathrm{~h}$ (section 4.2 ) we get a prediction for the core field strength at the surface of Io of about $5 \mathrm{nT}$. For the $42.95 \mathrm{~h}$ period the signal strength would be only $0.2 \mathrm{nT}$. A conductive core is detectable only for certain magma ocean configurations. A strong magma ocean will almost completely shield the core from the primary field. The maximum difference can be seen for weak magma oceans and for the solar rotation period. The primary field for this period is, however, very weak $(<0.01 \mathrm{nT})$. Mutual induction may in some cases (dark yellow in figure 7) allow for some contribution of a conductive core to the total signal even in the presence of a rather strong magma ocean. 


\subsubsection{Phase information}

The phase shift of the secondary field with respect to the primary field yields additional information for induction studies. A comparison of the panels of figure 7 and figure 8 shows, that while the amplitude rises monotonously toward the lower right corner of the displayed parameter space, the values for the phase shift reach a maximum value somewhere between the strong ocean case and the weak ocean case. Not so obvious but equally important are the different gradients of the isolines in some parts of the parameter space of figure 7 and figure 8. Therefore, two different internal configurations showing exactly the same amplitude of the induced field (lying on the same isoline in figure 7) may lead to different phase shifts and may be distinguished. Figure 8 shows that strong magma ocean configurations $(\sigma>1 \mathrm{~S} / \mathrm{m}, h>5 \mathrm{~km})$ suppress the phase shift almost completely. However, weaker magma oceans yield a significant phase shift of up to $70^{\circ}$. The timing of the observed induction signal is therefore another indicator for the conductivity of the satellite. The differences for the phase again show that a strong magma ocean shields the core. The maximum phase difference occurs for weak magma oceans and is only significant $\left(>10^{\circ}\right)$ for the orbital and solar rotation periods.

\subsection{Europa}

Europa's ocean is thought to lie beneath a relatively thin layer of ice (e.g. Pappalardo et al., 1999). The ocean is expected to be directly connected to the rocky mantle which may be one source of heat to keep it liquid (see e.g. Schubert et al., 2009). We use Europa models from Kuskov and Kronrod (2005) and again Zhang (2003). Both are four layer models including a FeSor Fe-core, a silicate mantle and a liquid ocean beneath an icy crust. The 
model parameters including our estimated conductivities are given in tables 6 and 7.

We display the amplitude as a function of ocean conductivity and thickness and the amplitude differences compared to the same model with a nonconductive core in figure 9.

\subsubsection{Observability of the ocean}

Both interior models show that a distinct ocean $(\sigma>1 \mathrm{~S} / \mathrm{m}, h>10 \mathrm{~km})$ induces signals which almost reach the primary field strength $(80 \%$ to $90 \%)$ for all periods. According to our results of section 4.2 such an ocean would produce signals at Europa's surface of $80 \mathrm{nT}$ at $11.23 \mathrm{~h}, 6 \mathrm{nT}$ at $85.22 \mathrm{~h}$ and $0.5 \mathrm{nT}$ at a period of $641.9 \mathrm{~h}$. The results for both models are almost indistinguishable. Obviously the slightly smaller ice shell thickness and the larger core conductivity of the Zhang (2003) model have no major influence. The nearly unconductive mantle layer does not affect the strength of the total signal. Up to a mantle conductivity of about $10^{-3} \mathrm{~S} / \mathrm{m}$ its influence lies beneath $1 \%$ of the primary signal. Our model predicts, however, significant signals of about $50 \%$ even for moderate ocean parameters of $h \approx 1 \mathrm{~km}$ and $\sigma \approx 5 \mathrm{~S} / \mathrm{m}$. Like other authors before (e.g. Zimmer et al., 2000, Schilling et al., 2007) we conclude that the large induction signals observed at Europa might very well be the result of a liquid ocean.

There are still many unknown parameters of the ocean like its depth, its extension, its salinity and so on. This information may be gained by obtaining magnetic field data of sufficient accuracy to be analyzed for multiple inducing frequencies. The measured amplitude for a single frequency can be explained by models with various ocean thicknesses $h$ and conductivities $\sigma$ 
(represented by isolines in figure 9). The amplitudes depend non-linearly on the interior conductivity parameters. Therefore, with amplitude information for multiple frequencies, all interior models which can not explain these values simultaneously can be ruled out. Information for each single frequency therefore narrows the valid parameter range for $\sigma$ and $h$. Our results show that multi-frequency measurements at Europa should be possible at all three presented frequencies.

\subsubsection{Observability of the core}

In the absence of a conductive ocean $(\sigma<1 \mathrm{~S} / \mathrm{m}, h<1 \mathrm{~km})$ the secondary field strength drops to about $5 \%$ of the primary field for the Zhang (2003) model and 1.4\% to 4\% (depending on the frequency) for the Kuskov and Kronrod (2005) model. The residuals are signals induced in the core. Although their relative values are larger than at Io, the lower primary field amplitudes give rise to about the same secondary field strength at the surface of $5 \mathrm{nT}$ at a period of $11.23 \mathrm{~h}$. For a period of $85.22 \mathrm{~h}$ the signal strength is about $0.4 \mathrm{nT}$. The major limiting factor for the detection of these signals is the strong damping by Europa's ocean. There is, however, a certain parameter range of $\sigma$ and $h$ (yellow in figure 9) where the mutual induction effect allows for a core signal of $1 \%$ to $2 \%$ in the presence of the ocean. If an ocean is clearly visible in the signal for multiple frequencies but one frequency lacks these $1 \%$ to $2 \%$ of the predicted signal strength this could be interpreted as the contribution of a conductive core due to mutual induction. This fact could be exploited to detect the core in future measurements. For example, with an iron core like in the Zhang (2003) model a contribution of about $2 \mathrm{nT}$ to the total signal at a period of $11.23 \mathrm{~h}$ is reached for ocean 
parameters inside the yellow colored region of figure 9 (lower panel). We therefore conclude that it might be possible to detect a conductive core at Europa with the induction method though the signal will likely be rather small.

\subsubsection{Phase information}

The phase shift for strong ocean cases at Europa $(\sigma>1 \mathrm{~S} / \mathrm{m}, h>10 \mathrm{~km})$ is small for the synodical and orbital inducing frequencies (see figure 10). It is, however, about $10^{\circ}$ to $20^{\circ}$ larger for the solar rotation period. When we compare both interior models we see that the higher core conductivity of the Zhang (2003) model leads to a much smaller phase shift for weak oceans $(\sigma<1 \mathrm{~S} / \mathrm{m}, h<1 \mathrm{~km})$. The difference in the phase shift in the case of a non-conductive core is also large in the absence of a conductive ocean (see color contours at the top left corners in all panels of figure 10).

\subsection{Ganymede}

Ganymede is not only another candidate for a subsurface liquid ocean, but also possesses the only known dynamo field of a moon in the solar system. Information about Ganymede's ocean and core are therefore of great scientific value. In order to analyze the secondary field at Ganymede we adapt models by Kimura et al. (2009) and Zhang (2003). The parameters used to adapt these models are given in the tables 8 and 9. The model of Kimura et al. (2009) consists of five layers with an ocean located between the crust and a lower mantle ice layer. Basis of this model are numerical simulations of the thermal history of Ganymede. Several additional models based on calculations of the temperature, pressure and density with depth 
profiles constrained by gravity measurements by the Galileo spacecraft were presented by Sohl et al. (2002). The outermost layer for those models includes a phase transition from ice I to ice III, ice V and ice VI through the first $900 \mathrm{~km}$ of the satellite. The thicknesses of the silicate layer below the ice and the innermost core layer vary between $900 \mathrm{~km}$ to $1100 \mathrm{~km}$ and 834 $\mathrm{km}$ to $634 \mathrm{~km}$ respectively, depending on the density of the core. Except for weaker signals from the core layer due to the smaller core radius, the Sohl et al. (2002) models show results for the induced signals which are very similar to those we infer for the Kimura et al. (2009) model. Therefore, only notable differences in the induced fields for these models are discussed here. The second model we present in greater detail was given by Zhang (2003). Although this four layer model is probably too simple to realistically describe Ganymede's interior we present it here to give the possibility to compare our results for Ganymede with those for the other satellites. The model consists of an iron core, a rocky mantle and an icy crust at the bottom of which we include Ganymede's ocean. Please note that the assumption that the ocean lies deep inside the interior and has contact to the rocky mantle is not very realistic. Still, we use this setup to investigate at which depth the ocean produces a significant signal. Also the presented range for the thickness of the ocean may be greatly exaggerated.

\subsubsection{Observability of the ocean}

The amplitude structures for both Ganymede models look quite different (isolines in figure 11). For the Kimura et al. (2009) model the top of the ocean is relatively close to the surface. This causes large induced amplitudes of about $80 \%$ even for average values for the oceans conductivity $(\sigma>0.1 \mathrm{~S} / \mathrm{m})$ 
and thickness $(h>0.1 \mathrm{~km})$. The mantle contributes less than $1 \%$ to these signals for conductivities lower than $10^{-3} \mathrm{~S} / \mathrm{m}$. The corresponding secondary field at Ganymede's surface which follows from the results of section 4.2 has a strength of about $32 \mathrm{nT}$ for $10.53 \mathrm{~h}, 0.6 \mathrm{nT}$ for $171.7 \mathrm{~h}$ and $0.5 \mathrm{nT}$ for a period of $641.9 \mathrm{~h}$. For the Zhang (2003) model the relative amplitudes are considerably smaller (up to about 50\% less), except for large values of $h(>100 \mathrm{~km})$. The reason for this is that the upper boundary of the ocean lies deep inside the satellite for thin ocean layers. As the lower boundary is kept fixed, the ocean is close to the surface only for large ocean thicknesses. We conclude that an ocean which lies deep in the interior of Ganymede will produce significantly weaker induction signals. For realistic ocean depths of about $150 \mathrm{~km}$ (e.g. Spohn and Schubert, 2003) this effect should be rather small. One should, however, keep in mind that the detectability of all oceans presented in this study depends on the depth of its upper boundary.

\subsubsection{Observability of the core}

The internal dynamo field discovered at Ganymede (Kivelson et al., 1996a) indicates the existence of a layer of molten iron in the core region of the satellite. Additional information about Ganymede's core, might be gained from induction caused by different periods of the time varying external magnetic field. The core's contribution to the total field induced by the primary field (neglecting Ganymede's internal field) in absence of an ocean on Ganymede is about $5 \%$ of the primary field for the Kimura et al. (2009) model and about $2 \%$ for the Zhang (2003) model, where the core radius is about $300 \mathrm{~km}$ smaller. The Ganymede model given by Schubert et al. (2004), whose results are otherwise similar to the Kimura et al. (2009) model, also shows less 
distinct core signals of about $2.7 \%$ due to its smaller core radius. Without a conductive ocean the resulting secondary field at the surface has a strength of about $2 \mathrm{nT}$ at a period of $10.53 \mathrm{~h}$ for the Kimura et al. (2009) model. The signal induced in the core easily becomes obscured by the overlying ocean. The mutual induction effect gives rise to core signals of up to $0.8 \mathrm{nT}$ in some cases (yellow regions in figure 11). This signal strength is, however, very likely too small to allow for a reliable detection of the core especially in the presence of plasma interaction fields.

\subsubsection{Phase information}

The phase information gives a similar picture for both models (figure 12). A strong ocean configuration $(\sigma>10 \mathrm{~S} / \mathrm{m}, h>10 \mathrm{~km})$ suppresses the phase shift. The same applies for the case of weak ocean configurations where the remaining signal represents the influence of the core. Therefore, a weak signal with little phase shift could be interpreted as a signal of the core alone while a strong signal without phase lag indicates a significant contribution of an ocean. The parameter regime with the biggest phase shift seems to coincide with the regime where mutual induction plays a role. In summary it seems promising to include the phase information in analysis of real data.

\subsection{Callisto}

Similar to Europa, Callisto is considered to be a candidate for a subsurface liquid water ocean due to its interior magnetic field that was interpreted as an induction signal from a conductive layer (Khurana et al., 1998, Neubauer, 1998a, Kivelson et al., 1999, Zimmer et al., 2000). Callisto has a moderate normalized moment of inertia of $C / M R^{2}=0.35$ suggesting its interior is 
only partially differentiated (Anderson et al., 2001). We address this fact by adapting the model of Kuskov and Kronrod (2005) as a six layer representation (table 10) with larger conductivities toward the center. The ocean is assumed to lie beneath an icy crust and above a mantle divided in three sub layers. Callisto does not possess a core with a significant amount of iron in this model so that its conductivity is rather low. Sohl et al. (2002) presented several additional multilayer models of Callisto's interior constrained by Galileo gravity measurements. The authors present two models assuming different core compositions and densities (pure olivine-type rock with $3300 \mathrm{~kg} \mathrm{~m}^{-3}$ and a mixture of $50 \mathrm{wt} \%$ iron and $50 \mathrm{wt} \%$ olivine). In these models the outermost icy shell with a thickness of $660 \mathrm{~km}$ includes a phase transition from ice I to ice VI. The layer beneath consists of hydrated silicates or a rock-ice mixture with a shell thickness of $1150 \mathrm{~km}$ to $1300 \mathrm{~km}$ depending on the core density. The results we infer using the Sohl et al. (2002) models are quite similar to the ones presented for the Kuskov and Kronrod (2005) model in this section. The only notable difference comes from a slightly larger crust, i.e. ice I layer thickness (we adopt a value of $180 \mathrm{~km})$. Therefore, instead of explicitly discussing the results for the Sohl et al. (2002) models we present results for a model by Zhang (2003) (table 11). This model consists of four layers with a larger crustal thickness and a larger core density than the Kuskov and Kronrod (2005) model. We adapt this larger density by increasing the core conductivity. As already mentioned for Ganymede, the Zhang (2003) model with an ocean located deep in the interior in direct contact to the mantle and with such a large range of thicknesses is hardly realistic. It should therefore not be used as a stand alone 
case, but only for comparison with the more realistic model by Kuskov and Kronrod (2005) and with the models given by Zhang (2003) for the other Galilean moons. The results are shown in figure 13 and 14 .

\subsubsection{Observability of the ocean}

Both models show a distinct signal of up to $80 \%$ for the synodic rotation period even for average values of the conductivity $(>1 \mathrm{~S} / \mathrm{m})$ and thickness $(>1 \mathrm{~km})$. The model from Sohl et al. (2002) gives results similar to the ones shown for Kuskov and Kronrod (2005) in figure 13. Only for strong ocean configurations (lower right corner of the figure) the amplitude is about 2 to $4 \%$ smaller due to the larger crustal thickness. The contribution of the mantle layers to these values is less than $1 \%$ as long as their conductivities are lower than $10^{-4} \mathrm{~S} / \mathrm{m}$. Taking into account the results of section 4.2 , the strength of the secondary field for the $10.18 \mathrm{~h}$ period is $16 \mathrm{nT}$ at the surface of Callisto. The rotation period of Callisto is comparable to the solar rotation period. For both frequencies a signal of $70 \%$ to $80 \%$ can be expected for strong ocean cases $(\sigma>10 \mathrm{~S} / \mathrm{m}, h>10 \mathrm{~km})$. The corresponding secondary field strengths are $0.9 \mathrm{nT}$ for $400.55 \mathrm{~h}$ and $0.4 \mathrm{nT}$ for a period of $641.9 \mathrm{~h}$. To apply the multi-frequency approach at Callisto it is necessary to measure the magnetic field in the vicinity of the satellite very precisely and to determine the contribution from the plasma interaction.

\subsubsection{Observability of the core}

We chose a low conductivity for the core in the Kuskov and Kronrod (2005) model. Such a core has almost no influence on the total induction signal and will certainly not be detectable. The situation looks somewhat better 
for the Zhang (2003) model. However, contributions of 1\% (with mutual induction, yellow regions in figure 13) to $2 \%$ (without a conductive ocean) only yield a secondary field strength at the surface of $0.2 \mathrm{nT}$ to $0.4 \mathrm{nT}$ at a period of $10.18 \mathrm{~h}$. We conclude that it will not be possible to gain information about Callisto's core from induction measurements at any frequency.

\subsubsection{Phase information}

The model results for Callisto show that a strong ocean $(\sigma>10 \mathrm{~S} / \mathrm{m}, h>$ $10 \mathrm{~km}$ ) suppresses a phase shift of the signal. For a non-conductive ocean the weak overall conductivity of the Kuskov and Kronrod (2005) model leads to a phase lag of nearly $90^{\circ}$. The larger core conductivity of the Zhang (2003) model on the other hand suppresses the phase shift to some degree. Therefore, if there were no ocean on Callisto one could possibly gain information about the core from the phase shift.

\subsection{Mutual induction}

Throughout this section we repeatedly mentioned the effect of mutual induction. When we subtract the amplitude results of models with a weak core conductivity from our original models we get negative values for certain ocean thicknesses and conductivities. This means that a less conductive core can lead to a larger induction signal than a highly conductive core if a conductive ocean is present. The reason for this is as follows. The timevariable primary field ((1) in figure 15) induces a field inside the satellite's core ((3) in fig. 15). This field in turn is part of the total field the conductive ocean (blue layer in fig. 15) experiences. However, the field induced in the core tries to act against the primary field and therefore has an opposite sign. 
It induces a magnetic field inside the ocean which leads to a decrease of the total induction signal outside the satellite ((4) and (4') in fig. 15). This mutual induction between the core and the ocean is completely included in the theoretical description of section 5. It is only significant when the ocean layer is not conductive enough or too thin to completely shield the underlying material from the primary field. On the other hand it needs to be conductive enough to allow for induction from the core signal. That is why we see this effect in an intermediate parameter region in the plots of section 6.1 to 6.4 (white and yellow regions in the amplitude results of figure 7 to 13). Generally all fields induced at the satellites in turn induce magnetic fields in all conductive layers.

\section{Induction Studies by Spacecraft Missions}

Magnetometer data of the Galileo spacecraft are so far the only sources for induction measurements at the Galilean moons. Only a few flybys exist which provide measurements in the vicinity of the satellites for a short period of time. It is also very difficult to adequately separate magnetic fields from plasma interaction effects in these data. Therefore, those measurements are not well suited for multi-frequency induction analysis. Additional magnetometer measurements by future Jupiter system missions are therefore necessary to apply the multi-frequency induction method.

For multi-frequency induction studies it is preferable to use magnetometer data from a spacecraft orbiting the satellite rather then just flyby data. An orbiter could perform continuous measurements over a time of several excitation periods and thus provide an excellent temporal coverage. The op- 
timal geometry for such orbits would be a low polar orbit. A coverage of the whole satellite surface can be achieved by the rotation of the orbit in the east-west direction. As the secondary field strength decreases by $r^{-3}$ with distance to the surface, it is crucial to keep the orbits as low as possible. In addition, the internal field "seen" from a low polar orbit will contain only small plasma magnetic fields from the thin shell between the orbital envelope and the surface. To gain information about the secondary field contributions at Ganymede it is necessary to determine the moments of the internal field. As the internal field is expected to be temporally constant throughout the orbiting phase, it should be relatively straightforward to obtain its strength.

When the data is acquired during flybys the best geometry for the measurements also is low polar flyby. In this case it is more difficult to filter out the plasma interaction field. Precise modeling of each flyby is necessary to apply induction techniques. Finally it is of course necessary to obtain a good absolute accuracy as well as good long term stability of the magnetometer. The challenge for the magnetometer and mission engineers will therefore be to enable measurements with suitable precision (About $0.1 \mathrm{nT}$ to $1 \mathrm{nT}$ according to the results in this paper).

The Europa Jupiter System Mission (EJSM/Laplace) under consideration by NASA and ESA might be the next opportunity to perform these measurements. At the current stage of planning, EJSM/Laplace includes two spacecraft. While Io and Callisto will presumably be encountered during several targeted flybys only the two spacecraft will later in the mission go into orbit around Europa and Ganymede, respectively. 


\section{Conclusions}

We analyze fluctuations of the primary magnetic field at the Galilean moons for three different classes of periods: The synodic Jovian rotation period with respect to the satellites and fractions of these periods, the satellites' orbital periods and the solar rotation period. The strongest field variations occur at the synodic rotation period in the $\mathrm{B}_{r}$ and $\mathrm{B}_{\theta}$ component. During one orbit of the satellites the fields of these two components are nearly elliptically polarized. $\mathrm{B}_{\theta}$ remains relatively constant but harbors most of the signals with the longer orbital and solar rotation periods. These signals originate in the eccentricity of the satellites orbits and the field caused by the Chapman-Ferraro currents in the magnetopause. Strong solar wind conditions therefore lead to stronger inducing long period inducing fields.

We analyzed the inductive response for various internal models of all Galilean moons. According to our results ocean layers at all of the satellites can easily produce secondary fields of about $70 \%$ to $90 \%$ of the primary field strength for all periods. A conductive core contributes up to $2 \%$ to the total signal for Io and $4 \%$ to $5 \%$ at Europa and Ganymede for the synodic rotation period. However, the core will be shielded from the primary field in the presence of an ocean. However, the mutual induction between the core and the ocean might decrease the amplitude for one of the inducing frequencies, which allows for a small core contribution to the total signal under certain circumstances. At Callisto it will probably not be possible to detect signals from the core. Additional information about both the cores and the oceans of all satellites is provided by information about the phase shift of the secondary signals. 


\section{${ }_{996}$ References}

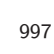
required data. this article. Edition.

To apply a multi-frequency approach using real magnetic data suitable measurements are necessary. To obtain a sufficient secondary signal strength, it would be preferable to perform precise magnetic measurements with a spacecraft on a low polar orbit. Such measurements would also help to determine the contribution from plasma interaction fields which otherwise obscure the signals at all satellites. The potential scientific value of these data is significant. The confirmation of liquid water and the characterization of the corresponding reservoirs at one of the Galilean moons as well as the possible implications regarding the origin of life and the formation of our solar system make it worthwhile to take all necessary efforts to gather the

\section{Acknowledgments}

These results were obtained within Schwerpunktprogramm "Planetary Magnetism" of die Deutsche Forschungsgemeinschaft under grant number SA 1772/3-1. We would like to thank Hauke Hussmann from the German Aerospace Center (DLR) for his advice concerning models of the interiors. We thank the NASA Planetary Data System for access to the Ulysses data used in this study. We also thank Alexandre Wennmacher for proof reading

Abramowitz, M., Stegun, I., 1964. Handbook of Mathematical Functions, 5th 
Alexeev, I. I., Belenkaya, E. S., 2005. Modeling of the Jovian Magnetosphere. Ann. Geophysicae 23, 809-826.

Anderson, J. D., Jacobson, R. A., McElrath, T. P., Moore, W. B., Schubert, G., Thomas, P. C., 2001. Shape, Mean Radius, Gravity Field, and Interior Structure of Callisto. Icarus 153, 157-161.

Anderson, J. D., Schubert, G., Jacobson, R. A., Lau, E. L., Moore, W. B., Sjogren, W. L., 1998. Europa's differentiated internal structure: Inferences from four Galileo encounters. Science 281, 2019-2022.

Beblo, M., Berktold, A., Bleil, U., Gebrande, H., Grauert, B., Haack, U., Haak, V., Kern, H., Miller, H., Petersen, N., Pohl, J., Rummel, F., Schopper, J. R., 1985. Physical Properties of Rocks. In: Landolt-Börnstein NewSeries, 6th Edition. Vol. V/1 Subvolume B. Springer-Verlag, pp. 1-604.

Bode, T. D., 1994. Modeling the Diurnally Precessing Jovian Magnetospheric Field. U.S.N.A. - Trident Scholar Project Report.

Carr, M. H., Belton, M. J. S., Chapman, C. R., Davies, M. E., Geissler, P., Greenberg, R., McEwen, A. S., Tufts, B. R., Greeley, R., Sullivan, R., Head, J. W., Pappalardo, R. T., Klaasen, K. P., Johnson, T. V., Kaufman, J., Senske, D., Moore, J., Neukum, G., Schubert, G., Burns, J. A., Thomas, P., Veverka, J., 1998. Evidence for a subsurface ocean on Europa. Nature $391,363-365$.

Chapman, S., Bartels, J., 1940. Geomagnetism. Vol. 2. Oxford Univ. Press. 
Chapman, S., Ferraro, V. C. A., 1930. A New Theory of Magnetic Storms. Nature 126, 129-130.

Connerney, J. E. P., Acuña, M. H., Ness, N. F., 1981. Modeling the Jovian current sheet and inner magnetosphere. J. Geophys. Res. 86, 8370-8384.

Connerney, J. E. P., Acuña, M. H., Ness, N. F., Satoh, T., 1998. New models of Jupiter's magnetic field constrained by the Io flux tube footprint. J. Geophys. Res. 103 (A6), 11929-11940.

Dobson, D. P., Brodholt, J. P., 2000. The Electrical Conductivity and Thermal Profile of the Earth's mid-mantle. Geophys. Res. Lett. 15 (27), 23252328.

Engle, I. M., 1992. Diurnal Variation in Jovian Subsolar Magnetopause Position. J. Geophys. Res. 97 (A11), 17169-17172.

Engle, I. M., Beard, D. B., 1980. Idealized Jovian Magnetosphere Shape and Field. J. Geophys. Res. A2 (85), 579-592.

Glassmeier, K., Klimushkin, D., Othmer, C., Mager, P., 2004. ULF waves at Mercury: Earth, the giants, and their little brother compared. Advances in Space Research 33, 1875-1883.

Glassmeier, K., Neubauer, F. M., Ness, N. F., Acuna, M. H., 1989. Standing hydromagnetic waves in the Io plasma torus - Voyager 1 observations. J. Geophys. Res. 94, 15063-15076.

Grimm, R. E., Stillman, D. E., Dec, S. F., Bullock, M. A., Priscu, J. C., 2007. 
Charge Mobility in Ice Brines and Ice-Silicate Mixtures. Annual report, NASA Exobiology Program.

Huddleston, D. E., Russell, C. T., Kivelson, M. G., Khurana, K. K., Bennett, L., 1998. Location and shape of the Jovian magnetopause and bow shock. J. Geophys. Res. 103 (E9), 20075-20082.

Jia, X., Kivelson, M. G., Khurana, K. K., Walker, R. J., 2009. Magnetic Fields of the Satellites of Jupiter and Saturn. Space Sci. Rev.

Keszthelyi, L., Jaeger, W. L., Turtle, E. P., Milazzo, M., Radebaugh, J., 2004. A post-Galileo view of Io's interior. Icarus 169, 271-286.

Keszthelyi, L., McEwen, A. S., Taylor, G. J., 1999. Revisiting the Hypothesis of a Mushy Global Magma Ocean in Io. Icarus 141, 415-419.

Khurana, K. K., 1997. Euler potential models of Jupiter's magnetospheric field. J. Geophys. Res. 102, 11295.

Khurana, K. K., Jia, X., Kivelson, M. G., Nimmo, F., Schubert, G., 2009. Evidence of a Global Magma Ocean in Io Revealed by Electromagnetic Induction. AGU Fall Meeting Abstracts, B6.

Khurana, K. K., Kivelson, M. G., Russel, C. T., 2002. Searching for Liquid Water in Europa by Using Surface Observatories. Astrobiology 2 (1), 93103.

Khurana, K. K., Kivelson, M. G., Stevenson, D. J., Schubert, G., Russell, C. T., Walker, R. J., Polanskey, C., 1998. Induced magnetic fields as evidence for subsurface oceans in Europa and Callisto. Nature 395, 777-780. 
Kimura, J., Nakagawa, T., Kurita, K., 2009. Size and compositional constraints of Ganymede's metallic core for driving an active dynamo. Icarus 202, 216-224.

Kivelson, M. G., Bagenal, F., Kurth, W. S., Neubauer, F. M., Paranicas, C., Saur, J., 2004. Magnetospheric interactions with satellites. In: Bagenal, F. (Ed.), Jupiter. The planet, satellites and magnetosphere. Cambridge Univ. Press, Ch. 21, pp. 513-536.

Kivelson, M. G., Khurana, K. K., Russell, C. T., Joy, S. P., Volwerk, M., Walker, R. J., Zimmer, C., Linker, J. A., 2001. Magnetized or unmagnetized: Ambiguity persists following Galileo's encounters with Io in 1999 and 2000. J. Geophys. Res. 106, 26121-26136.

Kivelson, M. G., Khurana, K. K., Russell, C. T., Volwerk, M., Walker, R. J., Zimmer, C., 2000. Galileo magnetometer measurements: A Stronger case for a subsurface ocean at Europa. Science 289, 1340-1343.

Kivelson, M. G., Khurana, K. K., Russell, C. T., Walker, R. J., Warnecke, J., Coroniti, F. V., Polanskey, C., Southwood, D. J., Schubert, G., 1996. Discovery of Ganymede's magnetic field by the Galileo spacecraft. Nature $384,537-541$.

Kivelson, M. G., Khurana, K. K., Russell, C. T., Walker, R. J., Warnecke, J., Coroniti, F. V., Polanskey, C., Southwood, D. J., Schubert, G., 1996a. Discovery of Ganymede's magnetic field by the Galileo spacecraft. Nature 384, 537-541. 
Kivelson, M. G., Khurana, K. K., Stevenson, D. J., Bennett, L., Joy, S., Russell, C. T., Walker, R. J., Zimmer, C., Polanskey, C., 1999. Europa and Callisto: Induced or intrinsic fields in a periodically varying plasma environment. J. Geophys. Res. 104 (A3), 4609-4625.

Kivelson, M. G., Khurana, K. K., Volwerk, M., 2002. The Permanent and Inductive Magnetic Moments of Ganymede. Icarus (157), 507-522.

Kivelson, M. G., Khurana, K. K., Walker, R. J., Warnecke, J., Russell, C. T., Linker, J. A., Southwood, D. J., Polanskey, C., 1996b. Io's Interaction with the Plasma Torus: Galileo Magnetometer Report. Science 274, 396-398.

Krupp, N., Vasyliunas, V. M., Woch, J., Lagg, A., Khurana, K. K., Kivelson, M. G., Mauk, B. H., Roelof, E. C., Williams, D. J., Krimigis, S. M., Kurth, W. S., Frank, L. A., Paterson, W. R., 2004. Dynamics of the Jovian magnetosphere. In: Jupiter. The Planet, Satellites and Magnetosphere. pp. 617-638.

Kuskov, O. L., Kronrod, V. A., 2005. Internal structure of Europa and Callisto. Icarus $177,550-569$.

Maumus, J., Bagdassarov, N., Schmeling, H., 2005. Electrical conductivity and partial melting of mafic rocks under pressure. Geochimica et Cosmochimica Acta 69 (19), 4703-4718.

Neubauer, F. M., 1980. Nonlinear standing Alfven wave current system at Io - Theory. J. Geophys. Res. 85, 1171-1178.

Neubauer, F. M., 1998a. Oceans inside Jupiter's moons. Nature 395, 749-750. 
Neubauer, F. M., 1998b. The sub-Alfvénic interaction of the Galilean satellites with the Jovian magnetosphere. J. Geophys. Res. 103 (E9), 1984319866.

Neubauer, F. M., 1999. Alfvén wings and electromagnetic induction in the interiors: Europa and Callisto. J. Geophys. Res. 104, 28671-28684.

Olsen, N., 1999. Induction studies with satellite data. Surveys in Geophysics 20, 309-340.

Pappalardo, R. T., Belton, M. J. S., Breneman, H. H., Carr, M. H., Chapman, C. R., Collins, G. C., Denk, T., Fagents, S., Geissler, P. E., Giese, B., Greeley, R., Greenberg, R., Head, J. W., Helfenstein, P., Hoppa, G., Kadel, S. D., Klaasen, K. P., Klemaszewski, J. E., Magee, K., McEwen, A. S., Moore, J. M., Moore, W. B., Neukum, G., Phillips, C. B., Prockter, L. M., Schubert, G., Senske, D. A., Sullivan, R. J., Tufts, B. R., Turtle, E. P., Wagner, R., Williams, K. K., 1999. Does Europa have a subsurface ocean? Evaluation of the geological evidence. J. Geophys. Res. 104, 24015-24056.

Parkinson, W., 1983. Introduction to Geomagnetism. Scottish Academic Press Ltd.

Parthasarathy, G., Sharma, S. R., 2004. High-temperature electrical and thermal properties of Burdett, Dalhart, Faucet and Wellman ordinary chondrites. Current Science 86 (10), 1366-1368.

Rathbun, J. A., Spencer, J. R., 2010. Ground-based observations of time variability in multiple active volcanoes on Io. Icarus 209, 625-630. 
Riley, K., Hobson, M., S.J. Bence, S., 2006. Mathematical Methods for Physics and Engineering, 3rd Edition. Cambridge Univ. Press.

Saur, J., 2004. A model of Io's local electric field for a combined Alfvénic and unipolar inductor far-field coupling. J. Geophys. Res. 109 (A18), 1210.

Saur, J., Neubauer, F. M., Glassmeier, K., 2010. Induced Magnetic Fields in Solar System Bodies. Space Sci. Rev. 152, 391-421.

Saur, J., Neubauer, F. M., Strobel, D. F., Summers, M. E., 2002. Interpretation of Galileo's Io plasma and field observations: 10, I24, and I27 flybys and close polar passes. J. Geophys. Res. 107, 1422.

Schilling, N., Neubauer, F. M., Saur, J., 2007. Time-varying interaction of Europa with the jovian magnetosphere: Constraints on the conductivity of Europa's subsurface ocean. Icarus 192, 41-55.

Schilling, N., Neubauer, F. M., Saur, J., 2008. Influence of the internally induced magnetic field on the plasma interaction of Europa. J. Geophys. Res. 113 (A12), 3203.

Schmucker, U., 1985. Magnetic and electric fields due to electromagnetic induction by external sources. In: Landolt-Börnstein New-Series. Vol. V/2 Subvolume B. Springer-Verlag, pp. 100-125.

Schubert, G., Anderson, J. D., Spohn, T., McKinnon, W. B., 2004. Interior composition, structure and dynamics of the Galilean satellites. In: Bagenal, F., Dowling, T. E., \& McKinnon, W. B. (Ed.), Jupiter. The Planet, Satellites and Magnetosphere. pp. 281-306. 
1151

1152

1153

1154

1155

1156

1157

1158

1159

1160

1161

1162

1163

1164

1165

1166

1167

1168

1169

1170

1171

Schubert, G., Sohl, F., Hussmann, H., 2009. Interior of Europa. In: Pappalardo, R. T., McKinnon, W. B., \& Khurana, K. K. (Ed.), Europa. p. 353.

Schubert, G., Spohn, T., Reynolds, R. T., 1986. Thermal histories, compositions and internal structures of the moons of the solar system. In: Burns, J. A., Mathews, M. S. (Eds.), Satellites. Univ. of Ariz. Press.

Sohl, F., Spohn, T., Breuer, D., Nagel, K., 2002. Implications from Galileo Observations on the Interior Structure and Chemistry of the Galilean Satellites. Icarus 157, 104-119.

Spohn, T., Schubert, G., 2003. Oceans in the icy Galilean satellites of Jupiter? Icarus 161, 456-467.

Stacey, F., 1992. Physics of the Earth. Brookfield Press, Brisbane.

Volwerk, M., Khurana, K., Kivelson, M., 2007. Europa's Alfvén wing: shrinkage and displacement influenced by an induced magnetic field. Annales Geophysicae 25, 905-914.

Waff, H. S., Weill, D. F., 1975. Electrical Conductivity of Magmatic Liquids: Effects of Temperature, Oxygen Fugacity and Composition. Earth and Planetary Science Letters 28, 254-260.

Wilson, R. J., Dougherty, M. K., 2000. Evidence Provided by Galileo of Ultra Low Frequency Waves Within Jupiter's Middle Magnetosphere. Geophys. Res. Lett. 27, 835-838. 
1172

1173

1174

1175

1176

Zhang, H., 2003. Internal Structure models and Dynamical Parameters of the Galilean Satellites. Cel. Mech. and Dynam. Astron. 87, 189-195.

Zimmer, C., Khurana, K. K., Kivelson, M. G., 2000. Subsurface oceans on Europa and Callisto: Constraints from Galileo magnetometer observations. Icarus 147, 329-347. 


\begin{tabular}{ccccc}
\hline & $\mathrm{a}\left[\mathrm{R}_{J}\right]$ & $\mathrm{T}[\mathrm{h}]$ & $\mathrm{i}[\mathrm{deg}]$ & $\mathrm{e}$ \\
\hline Io & 5.89 & 42.45 & 0.036 & 0.0041 \\
Europa & 9.38 & 85.22 & 0.469 & 0.0094 \\
Ganymede & 14.97 & 171.72 & 0.170 & 0.0011 \\
Callisto & 26.33 & 400.56 & 0.187 & 0.0074 \\
\hline
\end{tabular}

Table 1: Semi-major axis (a), orbital period (T), inclination (i) and eccentricity (e) of the Galilean moons, used in this work. 


\begin{tabular}{|c|c|c|c|c|c|c|c|c|c|}
\hline & \multicolumn{2}{|c|}{ Io } & \multicolumn{2}{|c|}{ Europa } & \multicolumn{2}{|c|}{ Ganymede } & \multicolumn{2}{|c|}{ Callisto } & \multirow{2}{*}{ Source } \\
\hline & $\mathrm{T}_{I}[\mathrm{~h}]$ & $\mathrm{A}_{I}[\mathrm{nT}]$ & $\mathrm{T}_{E}[\mathrm{~h}]$ & $\mathrm{A}_{E}[\mathrm{nT}]$ & $\mathrm{T}_{G}[\mathrm{~h}]$ & $\mathrm{A}_{G}[\mathrm{nT}]$ & $\mathrm{T}_{C}[\mathrm{~h}]$ & $\mathrm{A}_{C}[\mathrm{nT}]$ & \\
\hline \multirow{6}{*}{$\mathrm{B}_{r}$} & & & 3.74 & 1.2 & 3.51 & 3.3 & 3.39 & 5.9 & Current sheet \\
\hline & 4.32 & 16.1 & 3.74 & 1.6 & & & & & \\
\hline & 6.48 & 107.9 & 5.62 & 16.8 & 5.27 & 2.6 & 5.09 & & Quadrupole \\
\hline & 12.95 & 698.9 & 11.23 & 170.8 & 10.53 & 41.9 & 10.18 & 7.7 & Dipole \\
\hline & 12.95 & 50.9 & 11.23 & 45.8 & 10.53 & 43.9 & 10.18 & 36.1 & Current sheet \\
\hline & 42.45 & 2.9 & 85.22 & 10.5 & 171.70 & 1.7 & 400.55 & 0.7 & Inclination \\
\hline \multirow{4}{*}{$\mathrm{B}_{\Phi}$} & 4.32 & 12.1 & 3.74 & 1.2 & & & & & Octopole \\
\hline & 6.48 & 71.9 & 5.62 & 11.2 & 5.27 & & 5.09 & 0.2 & Quadrupole \\
\hline & 12.95 & 344.9 & 11.23 & 85.4 & 10.53 & 20.9 & 10.18 & 3.9 & Dipole \\
\hline & 12.95 & 32.5 & 11.23 & 19.1 & 10.53 & 9.7 & 10.18 & 6.9 & Current sheet \\
\hline \multirow{5}{*}{$\mathrm{B}_{\theta}$} & 6.48 & 13.0 & 5.62 & 1.3 & 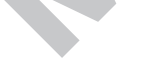 & & & & Octopole \\
\hline & 6.48 & 0.9 & 5.62 & & 5.27 & 3.0 & 5.09 & 1.9 & Current sheet \\
\hline & 12.95 & 103.1 & 11.23 & 16.0 & 10.53 & 2.5 & 10.18 & 0.3 & Quadrupole \\
\hline & 42.45 & 26.1 & & 15.8 & 171.70 & 1.0 & 400.55 & 0.5 & Eccentricity \\
\hline & 42.45 & 0.6 & 85.22 & 0.9 & 171.70 & 1.5 & 400.55 & 2.5 & Magnetopause \\
\hline
\end{tabular}




\begin{tabular}{|c|c|c|c|c|c|c|c|c|}
\hline 641.90 & 1.1 & 641.90 & 1.1 & 641.90 & 1.2 & 641.90 & 1.2 & MP variability \\
\hline$\left|\mathrm{B}_{p}\right|$ & 730 & & 300 & & 150 & & 400 & Plasma fields \\
\hline
\end{tabular}

Table 2: Major inducing signal amplitudes A (in nT) and periods $\mathrm{T}$ (in $\mathrm{h}$ ) for the Galilean moons for all magnetic components (System III coordinates). The underlying source of the contribution is indicated in the last column. The major inducing signals can be found in the $\mathrm{B}_{r}$ and $\mathrm{B}_{\Phi}$ components at the synodic Jovian rotation period. Long period signals mainly occur in the $\mathrm{B}_{\theta}$ component which has the weakest fluctuations. The bottom row shows the maximal plasma interaction fields for comparison. 


\begin{tabular}{|c|c|c|c|c|}
\hline Ref. & $\rho\left(\mathrm{g} / \mathrm{cm}^{3}\right)$ & $\sigma(\mathrm{S} / \mathrm{m})$ & Analogue & Source \\
\hline \multicolumn{5}{|c|}{ Silicate } \\
\hline (1) & 2.8 & $10^{-3}-10^{-5}$ & dry silicate & Beblo et al. (1985) \\
\hline$(2)$ & 2.9 & $10^{-3}-15$ & dry magma & Waff and Weill (1975),Beblo et al. (1985) \\
\hline$(3)$ & 3.1 & $10^{-2}$ & partially molten gabbro & Maumus et al. (2005) \\
\hline (8) & $3.5-3.7$ & $10^{-4}-10^{-10}$ & chondrites & Parthasarathy and Sharma (2004) \\
\hline$(10)$ & $3.2-3.4$ & $10^{-4}-10^{-8}$ & chondrites & Parthasarathy and Sharma (2004) \\
\hline \multicolumn{5}{|c|}{ Iron rich material } \\
\hline$(4)$ & 5.15 & $2-8$ & lower earth mantle & Dobson and Brodholt (2000) \\
\hline$(5)$ & 10 & $3 \times 10^{5}$ & outer earth core & Stacey (1992) \\
\hline (9) & 5 & 5 & lower earth mantle & Stacey (1992) \\
\hline \multicolumn{5}{|c|}{ Ice and water } \\
\hline (6) & 1 & $10^{-6}$ & $\mathrm{H}_{2} \mathrm{O}$ ice & Beblo et al. (1985) \\
\hline$(7)$ & 1 & $0.01-100$ & saline water & Beblo et al. (1985) \\
\hline$(11)$ & $2-3$ & $10^{-4}-10^{-6}$ & silicate $+41 \%$ water ice & Grimm et al. (2007) \\
\hline
\end{tabular}


Table 3: Sources of literature for the conductivity of the materials assumed in the adapted interior models of the Galilean moons. The index given in the first column refers to the indexes in the tables for the interior models (tables 4 to 11). Column 4 denotes the material from the literature which we use as an analog for the present material. 


\begin{tabular}{ccccc}
\hline & $h(\mathrm{~km})$ & $\sigma(\mathrm{S} / \mathrm{m})$ & $\rho\left(\mathrm{g} / \mathrm{cm}^{3}\right)$ & Ref. \\
\hline Crust & 25 & $10^{-4}$ & 2.8 & $(1)$ \\
Magma & $0-250$ & $10^{-3}-15$ & 2.9 & $(2)$ \\
Mantle & $996-1246$ & $10^{-2}$ & 3.1 & $(3)$ \\
FeS core & 550.6 & 5 & 5.15 & $(4)$ \\
\hline
\end{tabular}

Table 4: Io model adapted from Keszthelyi et al. (1999). The last column denotes the references for the sources of literature for the conductivity (see table 3). The thicknesses of the magma ocean and the mantle are varied, respectively. 


\begin{tabular}{ccccc}
\hline & $h(\mathrm{~km})$ & $\sigma(\mathrm{S} / \mathrm{m})$ & $\rho\left(\mathrm{g} / \mathrm{cm}^{3}\right)$ & Ref. \\
\hline Crust & 120 & $10^{-4}$ & 2.8 & $(1)$ \\
Magma & $0-500$ & $10^{-3}-15$ & 2.9 & $(2)$ \\
Mantle & $636-1136$ & $10^{-2}$ & 3.66 & $(3)$ \\
Fe core & 565.6 & $10^{5}$ & 8 & $(5)$ \\
\hline
\end{tabular}

Table 5: Io model adapted from Zhang (2003). References correspond to the literature given in table 3 . The thicknesses of the magma ocean and the mantle are varied, respectively. 


\begin{tabular}{ccccc}
\hline & $h(\mathrm{~km})$ & $\sigma(\mathrm{S} / \mathrm{m})$ & $\rho\left(\mathrm{g} / \mathrm{cm}^{3}\right)$ & Ref. \\
\hline Crust & $150-0$ & $2.1 \times 10^{-6}$ & 1 & $(6)$ \\
Ocean & $0-150$ & $0.01-100$ & 1 & $(7)$ \\
Mantle & 855 & $10^{-6}$ & 3.6 & $(8)$ \\
FeS core & 560 & 5 & 4.7 & $(9)$ \\
\hline
\end{tabular}

Table 6: Europa model adapted from Kuskov and Kronrod (2005). References correspond to the literature given in table 3 . The thicknesses of the ocean and the crust are varied, respectively. 


\begin{tabular}{ccccc}
\hline & $h(\mathrm{~km})$ & $\sigma(\mathrm{S} / \mathrm{m})$ & $\rho\left(\mathrm{g} / \mathrm{cm}^{3}\right)$ & Ref. \\
\hline Crust & $100-0$ & $10^{-6}$ & 1 & $(6)$ \\
Ocean & $0-100$ & $0.01-100$ & 1 & $(7)$ \\
Mantle & 865 & $10^{-5}$ & 3.13 & $(10)$ \\
Fe core & 596 & $10^{5}$ & 8 & $(5)$ \\
\hline
\end{tabular}

Table 7: Europa model adapted from Zhang (2003). References correspond to the literature given in table 3 . The thicknesses of the ocean and the crust are yaried, respectively. 


\begin{tabular}{ccccc}
\hline & $h(\mathrm{~km})$ & $\sigma(\mathrm{S} / \mathrm{m})$ & $\rho\left(\mathrm{g} / \mathrm{cm}^{3}\right)$ & Ref. \\
\hline Crust & 150 & $10^{-6}$ & 1 & $(6)$ \\
Ocean & $500-0$ & $0.01-100$ & 1 & $(7)$ \\
Icy mantle & $350-850$ & $10^{-6}$ & 1.8 & $(11)$ \\
Mantle & 650 & $10^{-5}$ & 3.5 & $(8)$ \\
Core & 981.2 & $10^{5}$ & 8 & $(5)$ \\
\hline
\end{tabular}

Table 8: Ganymede model adapted from Kimura et al. (2009). References correspond to the literature given in table 3. The thicknesses of the ocean and the icy mantle are varied, respectively. 


\begin{tabular}{ccccc}
\hline & $h(\mathrm{~km})$ & $\sigma(\mathrm{S} / \mathrm{m})$ & $\rho\left(\mathrm{g} / \mathrm{cm}^{3}\right)$ & Ref. \\
\hline Crust & $785-0$ & $10^{-6}$ & 1 & $(6)$ \\
Ocean & $0-785$ & $0.01-100$ & 1 & $(7)$ \\
Mantle & 1136 & $10^{-5}$ & 3.1 & $(10)$ \\
Core & 710.2 & $10^{5}$ & 8 & $(5)$ \\
\hline
\end{tabular}

Table 9: Ganymede model adapted from Zhang (2003). References correspond to the literature given in table 3 . The thicknesses of the ocean and the crust are varied, respectively. 


\begin{tabular}{ccccc}
\hline & $h(\mathrm{~km})$ & $\sigma(\mathrm{S} / \mathrm{m})$ & $\rho\left(\mathrm{g} / \mathrm{cm}^{3}\right)$ & Ref. \\
\hline Crust & 150 & $10^{-6}$ & 1 & $(6)$ \\
Ocean & $0-450$ & $0.01-100$ & 1 & $(7)$ \\
Mantle 1 & $450-0$ & $10^{-6}$ & 2 & $(11)$ \\
Mantle 2 & 300 & $10^{-5}$ & 2.15 & $(11)$ \\
Mantle 3 & 910 & $10^{-4}$ & 2.43 & $(11)$ \\
Core & 600.3 & $10^{-2}$ & 3.15 & $(3)$ \\
\hline
\end{tabular}

Table 10: Callisto model adapted from Kuskov and Kronrod (2005). References correspond to the literature given in table 3 . The thicknesses of the ocean and the mantle are varied, respectively. 


\begin{tabular}{ccccc}
\hline & $h(\mathrm{~km})$ & $\sigma(\mathrm{S} / \mathrm{m})$ & $\rho\left(\mathrm{g} / \mathrm{cm}^{3}\right)$ & Ref. \\
\hline Crust & 200 & $10^{-6}$ & 1 & $(6)$ \\
Ocean & $0-1000$ & $0.01-100$ & 1 & $(7)$ \\
Mantle & $1439-439$ & $10^{-5}$ & 2.15 & $(11)$ \\
Core & 771.3 & 5 & 4.5 & $(4)$ \\
\hline
\end{tabular}

Table 11: Callisto model adapted from Zhang (2003). References correspond to the literature given in table 3 . The thicknesses of the ocean and the mantle are varied, respectively. 
Figure 1: Field lines for the superposition of (A) the VIP4 model and the Khurana (1997) current sheet, (B) the VIP4, the Connerney et al. (1981) current sheet and the Engle (1992) no tilt magnetopause model for $\mathrm{R}_{s s}=60 \mathrm{R}_{J}$ plotted at $\Phi=292^{\circ} / 112^{\circ}$ System III longitude. The x-axis points sunwards, the z-axis north. 
Figure 2: Data for (A) the velocity and (B) the magnetopause distance $R_{s s}$ calculated from Ulysses data from the second half of the year 1992. The velocity shows a clear periodicity of about 27 days. The values of $R_{s s}$ lie in a realistic range for $R_{s s}$ with an average of about $70 R_{J}$ (dashed line). 
Figure 3: $\mathrm{B}_{r}$ vs. $\mathrm{B}_{\Phi}$ along the satellites' orbits from a superposition of the models mentioned in section 3. The field is elliptically polarized. Eccentricity and inclination of the orbits lead to a broadening of the lines. 
Figure 4: Total amplitude spectra for Io, Europa, Ganymede and Callisto. Arrows indicate the synodical period, the orbital period and the solar rotation period, respectively. Note that the scale of the y-axis varies from panel to panel. The displayed amplitudes may differ from the more precise values given in table 2 due to losses by spectral leakage. The length of the analyzed time series is $5,100 \mathrm{~h}$ sampled by 41,000 data points. 
Figure 5: Peak amplitude of the magnetopause field's $\mathrm{B}_{\theta}$ component at the orbital period as a function of the subsolar point distance $\mathrm{R}_{s s}$ for all Galilean moons. 
Figure 6: Concept of a spherical conductivity distribution for $J$ shells.

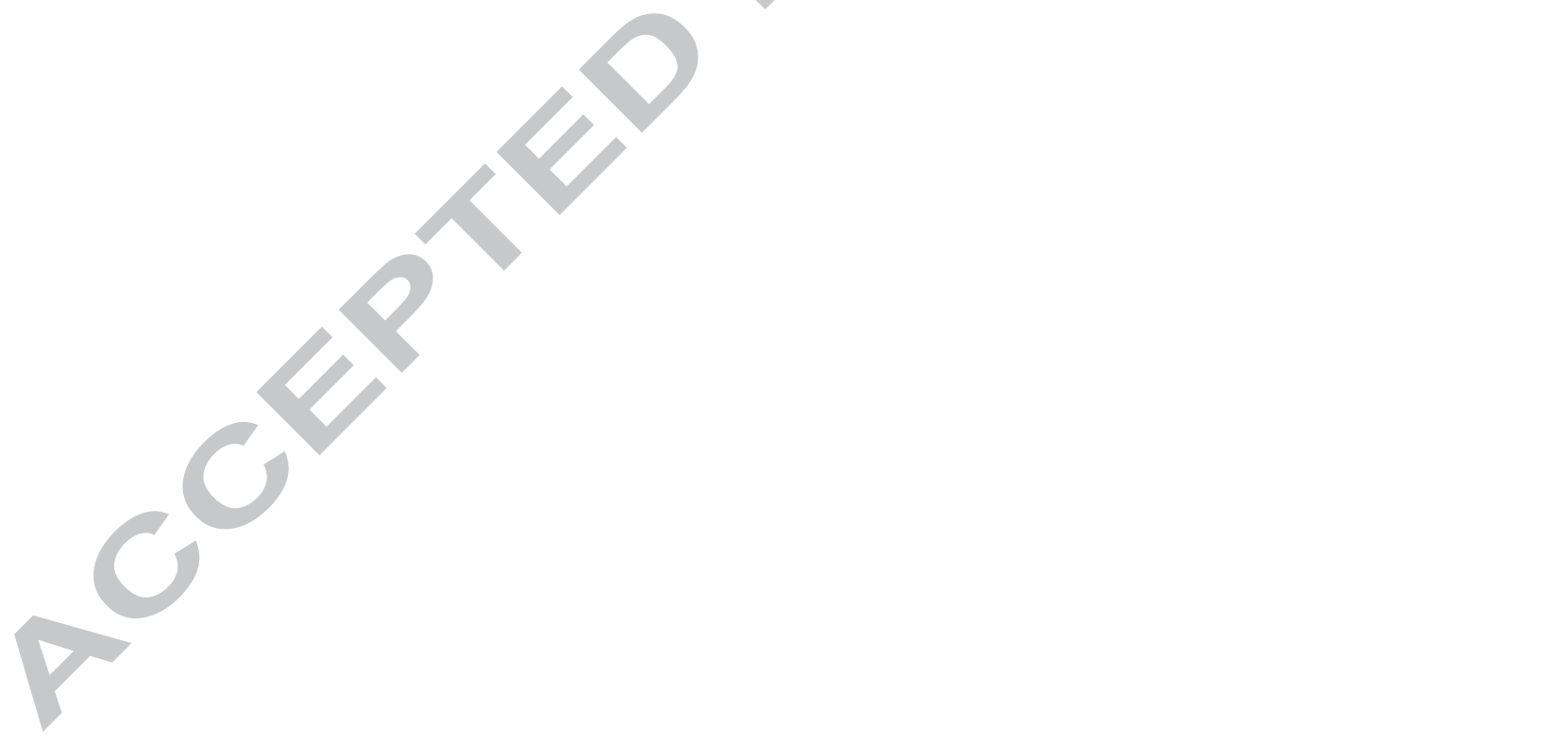


Figure 7: Amplitudes of the induced field for the Io-Keszthelyi et al. (1999) model (upper panel) and the Io-Zhang (2003) model (lower panel) for three primary field periods $\mathrm{T}$ and various magma ocean thicknesses and conductivities. Isolines: induced amplitude in $\%$ of the primary field strength. Color encoded: differences of the induced amplitudes $\Delta \mathrm{A}$ of interior models with and without a conductive core in $\%$ of the primary field strength. White and yellow areas indicate negative values caused by the mutual induction between ocean and core. 
Figure 8: Phase shift of the secondary field with respect to the primary field for the IoKeszthelyi et al. (1999) model (upper panel) and the Io-Zhang (2003) model (lower panel) for three primary field periods $\mathrm{T}$ and various magma ocean thicknesses and conductivities. Isolines: phase shift relative to the primary field in degrees. Color encoded: differences of the phase shifts $\Delta \Phi$ of interior models with and without a conductive core in degrees. 
Figure 9: Amplitudes of the induced field for the Europa-Kuskov and Kronrod (2005) model (upper panel) and the Europa-Zhang (2003) model (lower panel) for three primary field periods $\mathrm{T}$ and various ocean thicknesses and conductivities. Isolines: induced amplitude in $\%$ of the primary field strength. Color encoded: differences of the induced amplitudes $\Delta \mathrm{A}$ of interior models with and without a conductive core in $\%$ of the primary field strength. White and yellow areas indicate negative values caused by the mutual induction between ocean and core. 
Figure 10: Phase shift of the secondary field with respect to the primary field for the Europa-Kuskov and Kronrod (2005) model (upper panel) and the Europa-Zhang (2003) model (lower panel) for three primary field periods $\mathrm{T}$ and various ocean thicknesses and conductivities. Isolines: phase shift relative to the primary field in degrees. Color encoded: differences of the phase shifts $\Delta \Phi$ of interior models with and without a conductive core in degrees. 
Figure 11: Amplitudes of the induced field for the Ganymede-Kimura et al. (2009) model (upper row) and the Ganymede-Zhang (2003) model (lower row) for three primary field periods $\mathrm{T}$ and various ocean thicknesses and conductivities. Isolines: induced amplitude in $\%$ of the primary field strength. Color encoded: differences of the induced amplitudes $\Delta \mathrm{A}$ of interior models with and without a conductive core in $\%$ of the primary field strength. White and yellow areas indicate negative values caused by the mutual induction between ocean and core. 
Figure 12: Phase shift of the secondary field with respect to the primary field for the Ganymede-Kimura et al. (2009) model (upper row) and the Ganymede-Zhang (2003) model (lower row) for three primary field periods $\mathrm{T}$ and various ocean thicknesses and conductivities. Isolines: phase shift relative to the primary field in degrees. Color encoded: differences of the phase shifts $\Delta \Phi$ of interior models with and without a conductive core in degrees. 
Figure 13: Amplitudes of the induced field for the Callisto-Kuskov and Kronrod (2005) model (upper row) and the Callisto-Zhang (2003) model (lower row) for three primary field periods $\mathrm{T}$ and various ocean thicknesses and conductivities. Isolines: induced amplitude in $\%$ of the primary field strength. Color encoded: differences of the induced amplitudes $\Delta \mathrm{A}$ of interior models with and without a conductive core in $\%$ of the primary field strength. White and yellow areas indicate negative values caused by the mutual induction between ocean and core. 
Figure 14: Phase shift of the secondary field with respect to the primary field for the Callisto-Kuskov and Kronrod (2005) model (upper row) and the Callisto-Zhang (2003) model (lower row) for three primary field periods $\mathrm{T}$ and various ocean thicknesses and conductivities. Isolines: phase shift relative to the primary field in degrees. Color encoded: differences of the phase shifts $\Delta \Phi$ of interior models with and without a conductive core in degrees. 
Figure 15: Concept of the mutual induction effect at a satellite with two conductive layers (e.g. a conductive ocean $\sigma_{J-1}$ and core $\sigma_{1}$ ). Black arrow: Primary field (1), blue arrow: secondary ocean field (2) induced by (1), red arrow: secondary core field (3) induced by (1), green arrow: ocean field (4) induced by (3). (2'), (3') and (4') represent the induced fields as they would be measured at the surface of the satellite. For suitable internal structures (3') becomes smaller than $\left(4^{\prime}\right)$ and the mutual induction reduces the total signal. 


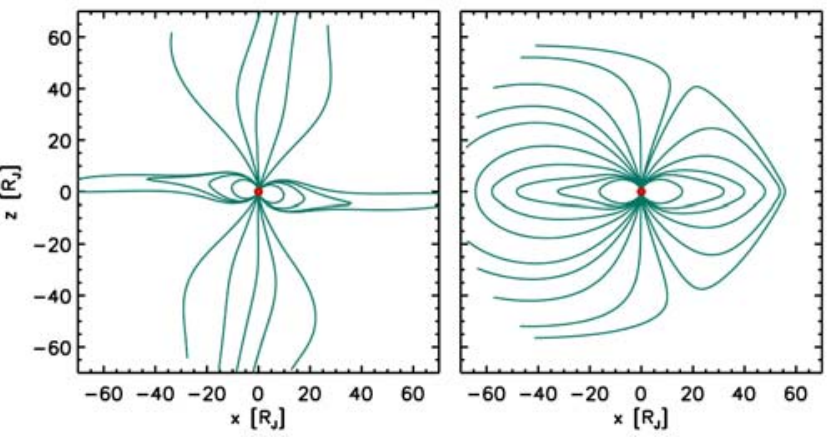




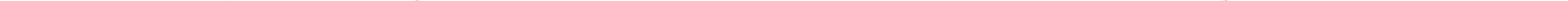




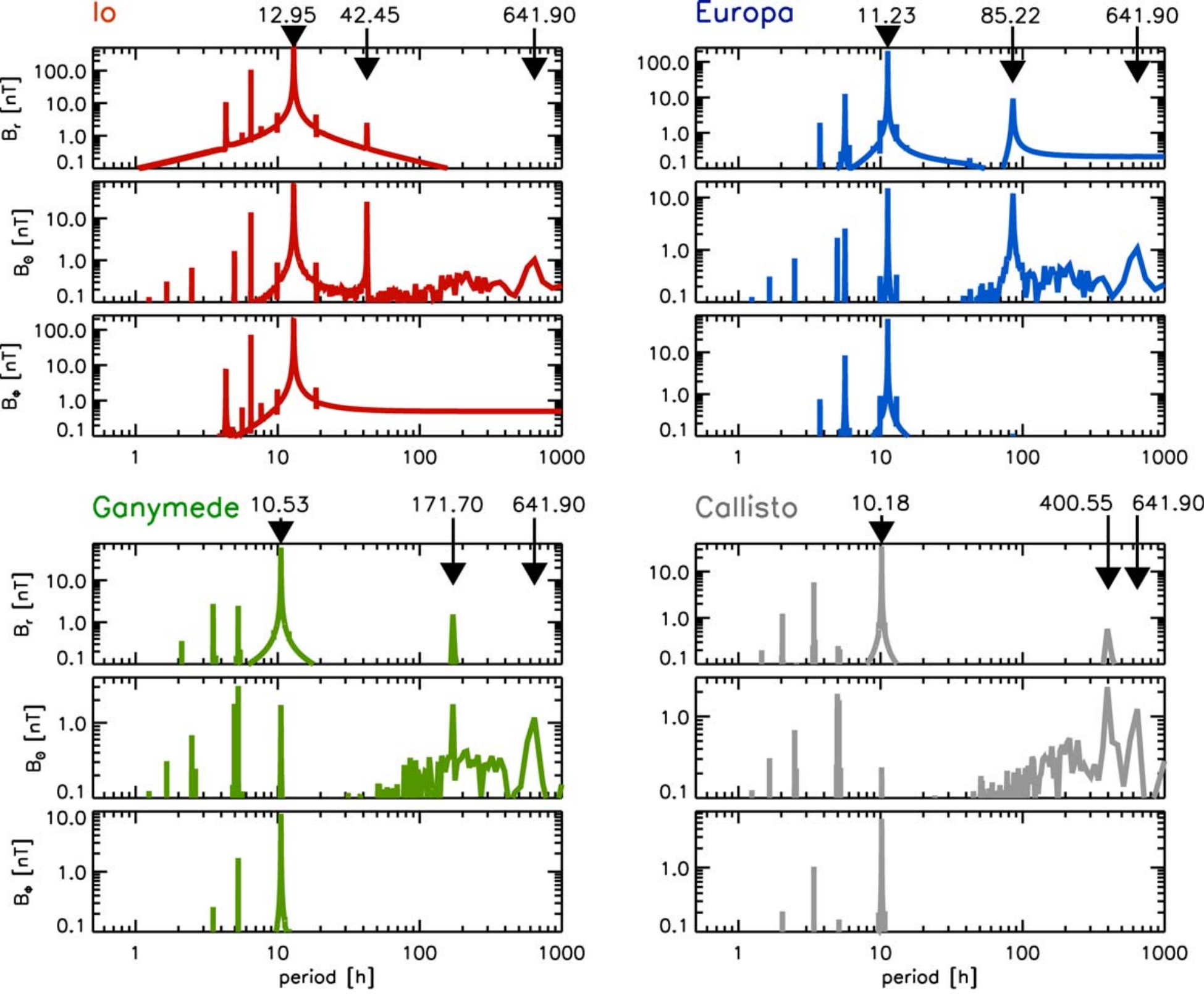




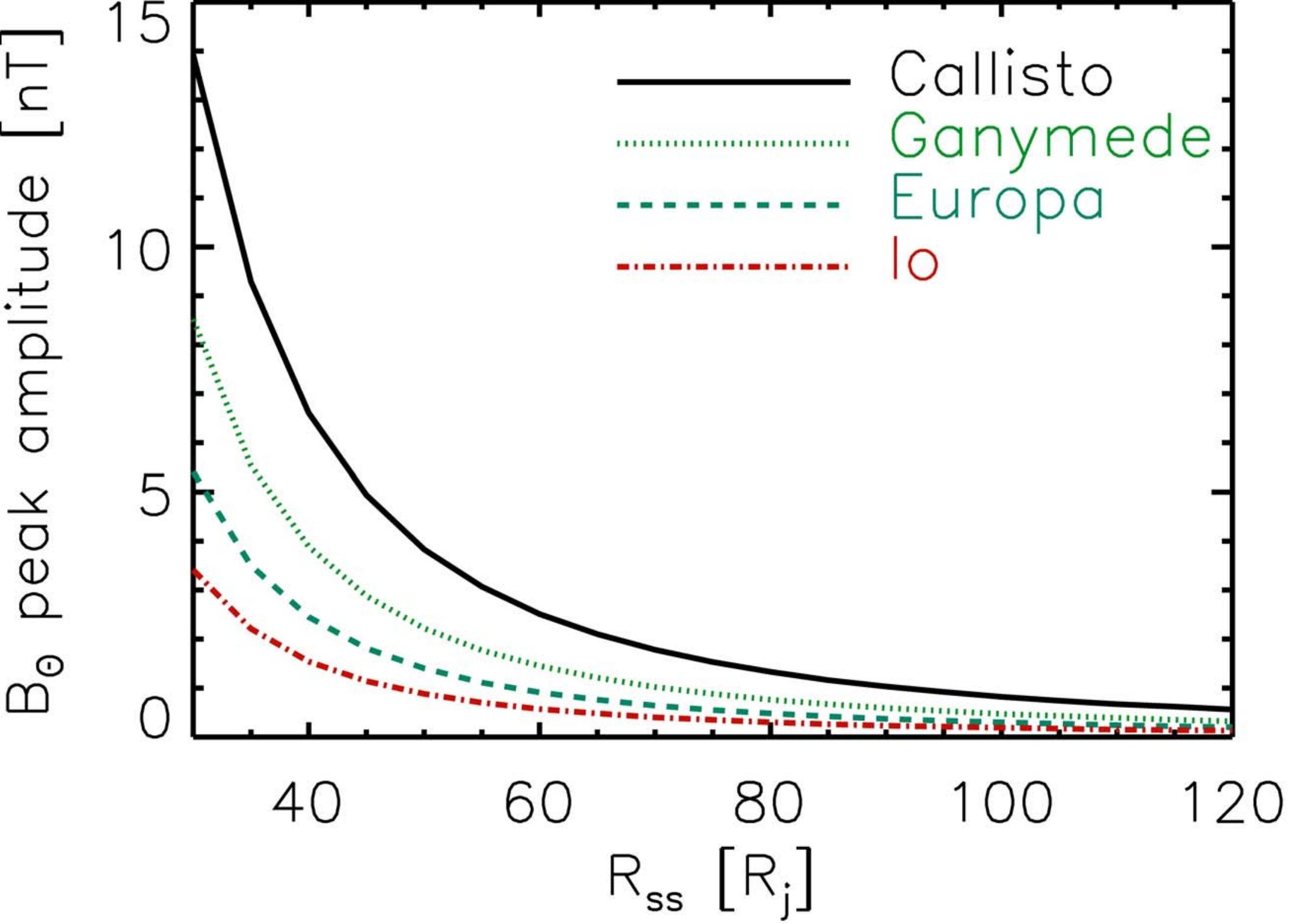




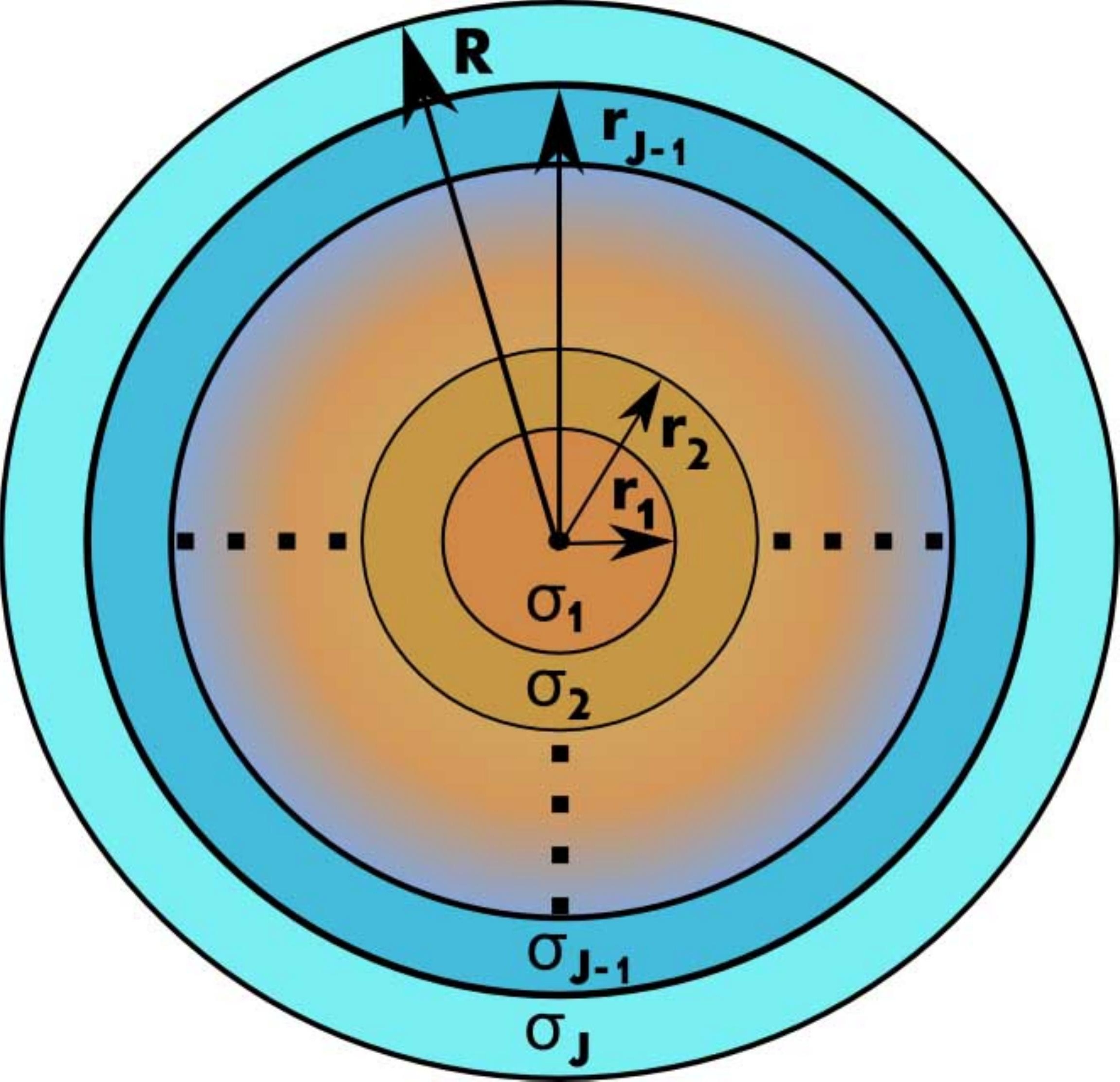




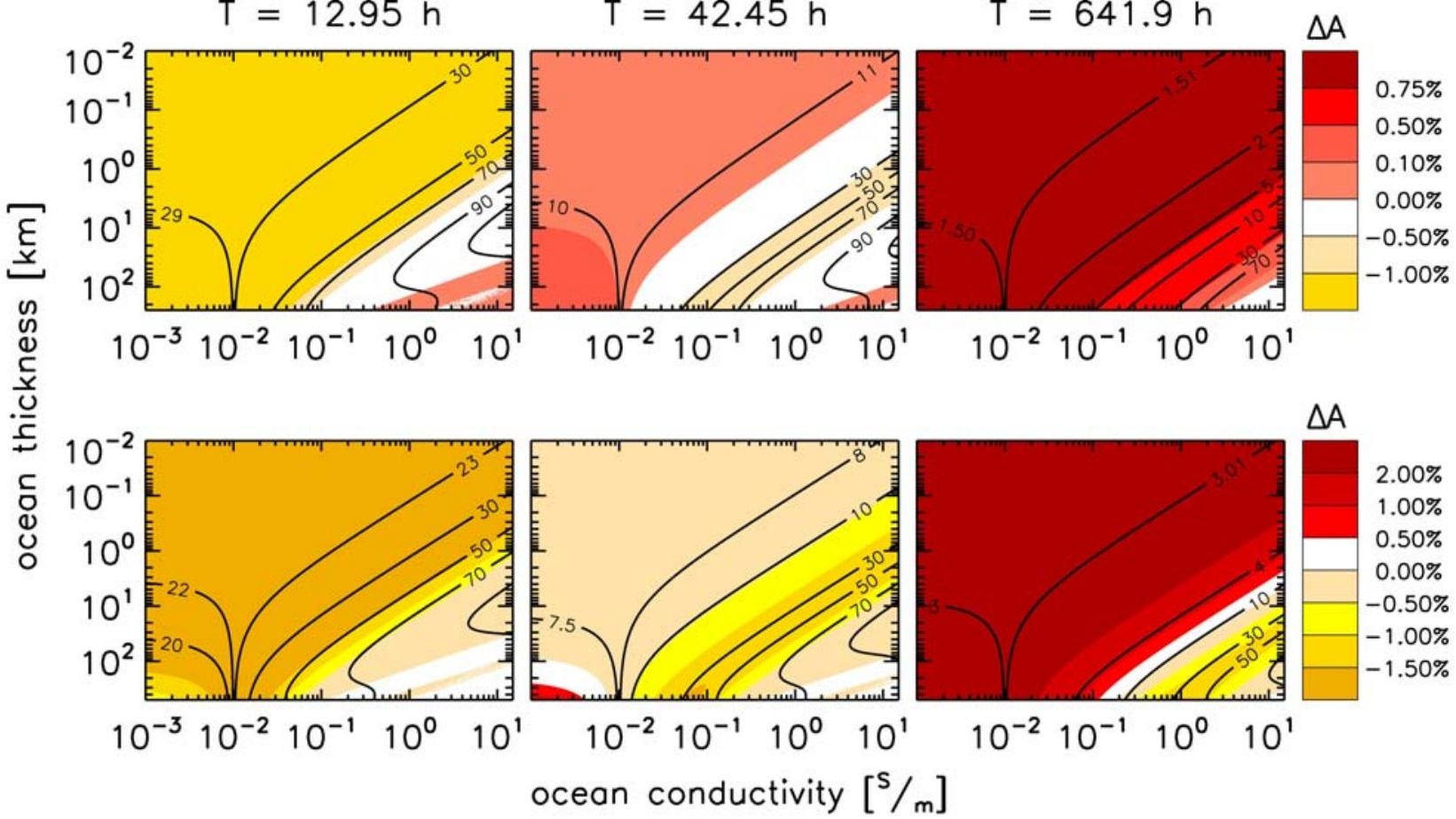




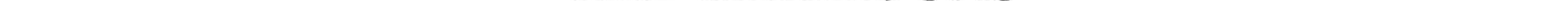




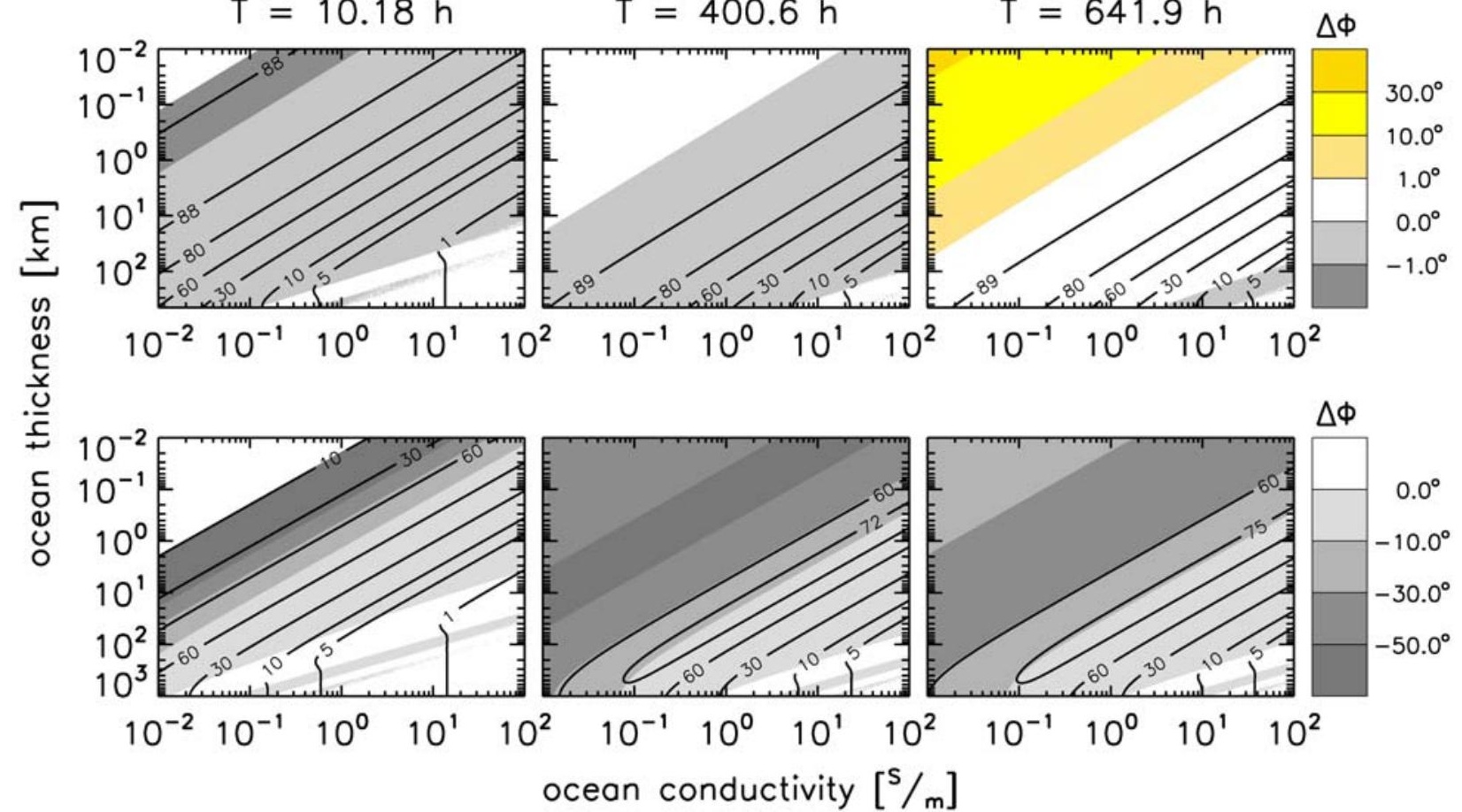


fields measured at the surface:
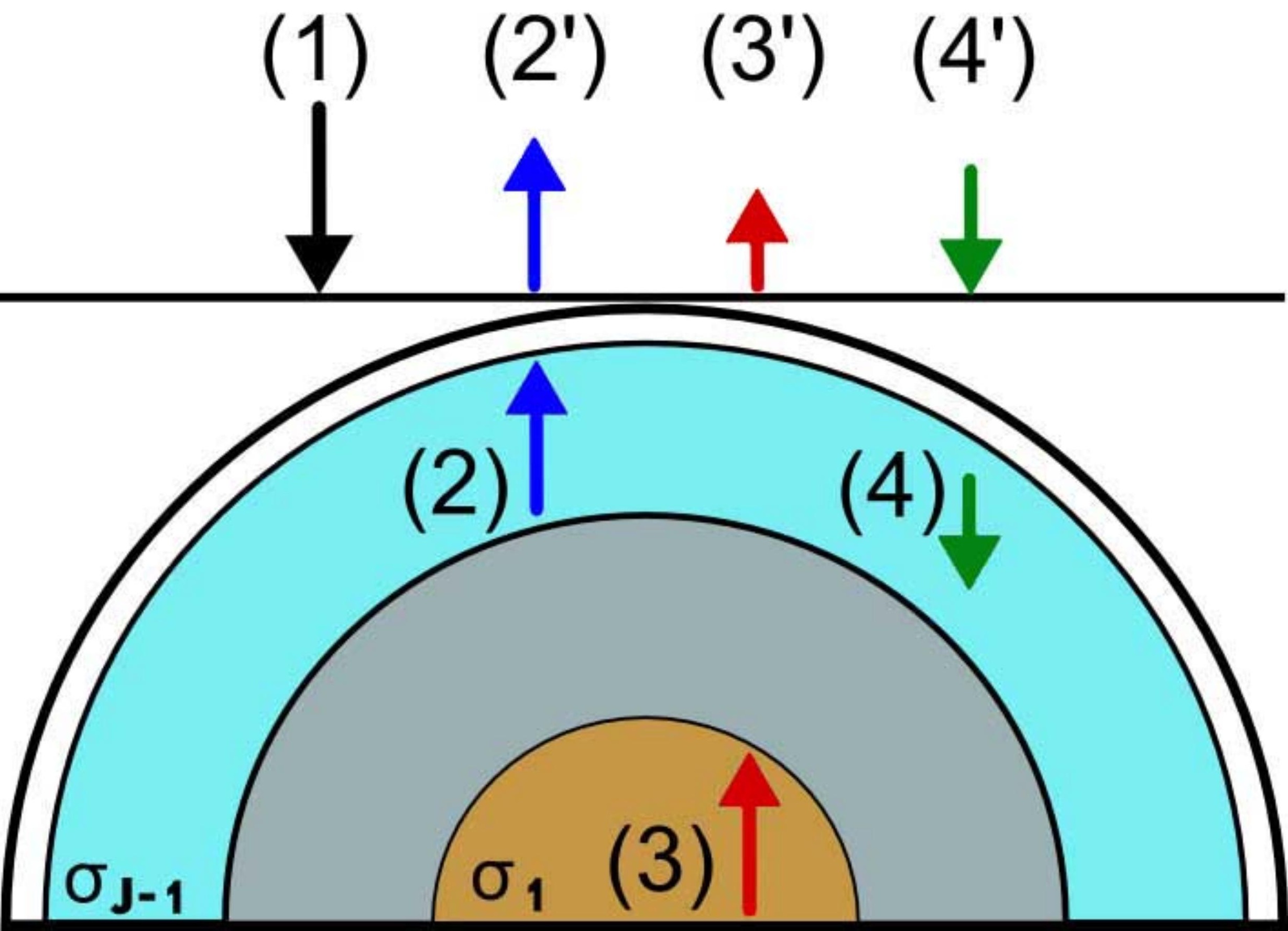
- We derive and discuss inducing magnetic field signals of various frequencies inside the Jovian magnetosphere

- Inductive responses of the interiors of the Galilean satellites are discussed

- Signals originating from subsurface oceans should be detectable

- The core layers will be very hard to detect 